\title{
Biostratigraphic constraints
}

\section{KATWAZ BASIN}

Age of the Katawaz Basin as given in figures 3 and 7 of Carter et al. (this paper) is based on Qayyum (1997), Qayyum et al. (2001) and our new data. All data, except for Qayyum et al. (2001), which is readily accessible in the open literature, is given below.

A) Extract from Qayyum, M, 1997, Sedimentation and tectonics of the Tertiary Katawaz Basin, NW Pakistan: a basin analysis approach. Unpublished PhD thesis, Corvallis, Oregon State University, USA, 480p.

B) New Nannofossil data provided for this paper by co-author Paul Bown (UCL, London), from samples collected by Qayyum et al. (2001).

\section{A) Age of Nisai Formation (Qayyum 1997 - extract from PhD thesis).}

The Nisai Formation contains both planktonic and benthic foraminifers, gastropods, pelecypods, and echinoderms. On the basis of the faunal assemblages, Jones (1961) assigned an early Eocene to early Oligocene age to the Nisai Limestone in the Katawaz Basin. Allemann (1979) studied this unit farther to the east in the vicinity of Muslimbagh, where this unit unconformably overlies ophiolites. He suggested that the oldest carbonate strata overlying the ophiolites are lower Eocene to lower middle Eocene.

The Nisai Formation was extensively sampled for during the measurement of these sections. Dr. Neil Wells of Kent State University has identified (written commun.) several genera and species of benthic forams, some of these are new. The foraminiferal assemblage Assilina dandotica, Discocyclina ranikotensis, Miscellanea stampi, and Operculina subsalsa indicate that the oldest age of the Nisai in the Katawaz Basin is earliest late Paleocene. Other notable Paleocene foraminifera is Saudia labyrinthica. Lockhartia hunti var. pustulosa, Nummulites dolloto, and Assilina granulosa (placentula), which suggest an early Eocene age. Assilina granulosa, A. granulosa var. spinosa, A. laminosa, Nummulites atacicus/dolloti, and Orbitolites complanatus indicate an early to middle Eocene age. Alveolina elliptica is typical of the middle Eocene. A few species also indicate a late Eocene age. Only two samples, one from the Gardab Manda section and the other from the Rud Faqirzai section, contain Nummulites fichteli, which suggests a possible early Oligocene age. The sample from the Rud Faqirzai section is from rare foram-bearing debris flow deposit in the basal part of siliciclastic Murgha Faqirzai member. The other fossil identified from the same sample was Kathina selveri, which is possibly of Paleocene age.

B) New nannofossil data analysed by co-author $P$. Bown, from samples collected by Qayyum et al for Qayyum et al (2001). 


\section{Sample 91MZ-27. Nisai Fm. Rud Fakirzai section}

Common moderately preserved nannofossils.

Nannofossil biozone NP13 (49.5-50.7 Ma) lower Early Eocene.

Discoaster keupperi

Discoaster lodoensis

Helicosphaera sp.

No Tribrachiatus orthostylus

No Discoaster sublodoensis

\section{Sample 91N-439 Nisai Fm. Nisai Railroad section}

Few moderately preserved nannofossils.

Nannofossil biozones NP17-20 upper middle Eocene-upper Eocene (34.4-40.4 Ma)

Reticulofenestra reticulata

Reticulofenestra scripssae

Reticulofenestra lockeri

Pemma sp.

Discoaster saipanensis

\section{Sample 91MZ-102 Nisai Fm. Nisai Rairoad section}

Rare poor to moderately preserved nannofossils

Nannofossil biozone NP18? upper Eocene ( 36-37 Ma)

Reticulofenestra scripssae

Cyclicargolithus floridanus

Sphenolithus moriformis

Sphenolithus radians

Calcidiscus protoannulus

Reticulofenestra cf. $R$. reticulata

Coccolithus pelagicus

Rosette discoaster indet.

Daktylethra sp. indet.

Zygrhablithus bijugatus?

Helicosphaera compacta/clarissima

\section{Sample 91N-349 Shaigalu Fm. Rud Faqirzai section}

Rare M

Middle Eocene-lower Miocene

Cyclicargolithus floridanus

Sphenolithus moriformis

Micula staurophora (Cretaceous reworking)

Watznaueria barnesiae (Cretaceous reworking)

\section{N-38 Shaigalu. Manzaki Kili 1 section.}

Very rare nannofossils. 
Cretaceous (reworking)

Eiffellithus eximius

Tranolithus minimus

Tranolithus orionatus

Watznaueria barnesiae

Watznaueria britannica

Sample 91RL-35. Shaigalu Formation.Manzaki Kili 2 section.

Very rare nannofossils.

Cretaceous (reworking)

Micula staurophora

Prediscosphaera cretacea

Watznaueria barnesiae

Sample 91N-9 Shaigalu Fm. Manzaki Kili 1.

Barren of nannofossils

Sample 91N-316 Murgha Fakirzai Fm. Rud Faqirzai section.

Barren of nannofossils

Sample 91MZ-50

Barren of nannofossils

SUMMARY: Overall these data imply that the age of the Nisai Formation ranges from late Paleocene to early Oligocene. However, relative abundance of pre-Oligocene fossils suggests that most of the formation was deposited from late Paleocene to late Eocene. An early Oligocene age for the uppermost Nisai Formation is used as the baseline for the deposition of the overlying Khojak Formation. Sample depositional ages within the Khojak Formation are assigned according to their position within the stratigraphic sections (shown in Qayyum et al. (1996) and the biostratigraphic age ranges reported by Qayyum et al. (2001).

\section{References}

Allemann, F., 1979, Time of emplacement of the Zhob valley ophiolites and Belta ophiolites, Baluchistan (Preliminary report) in Farah, A. and Dejong, K.A. (eds), Geodynamics of Pakistan: Quetta, Geological Survey of Pakistan p 215-242.

Jones, A.G. (ed), 1961, Reconnaissance geology of part of west Pakistan. A Colombo Plan Cooperative Project. Toronto, Government of Canada, Hunting Survey Corporation, $550 \mathrm{p}$. 


\section{MAKRAN}

To ensure age control samples were collected from type locations described in the Geological Survey of Iran Area Report No. 57, 1985 (North Makran \& South Baluchestan). This report details the biostratigraphic data used for age control and the relevant data are summarised below. A review of the data has not changed the original ages assigned in 1985. We were unable to find nannofossils in the sampled rocks to place tighter age control. Without such data the stratigraphic age range forms the depositional age uncertainty assigned to each sampled unit.

\section{PISHIN UNIT (N4 Early Miocene)}

Outer neritic depositional environment

Planktonic foraminifera

Globoratalia kugleri

Globoratalia continuosa

Globoratalia siakensis

Globigerina venezuelana

Globigerina ciperoensis

Globigerina praebulloides

Globigerinoides trilobus

Globigerinoides sicanus

Globigerinoides sacculifer

Globigerinoides subquadratus

Globaoquadrina altispira altispira

Praeorbulina glomerosa

$$
\begin{gathered}
\text { Benthics } \\
\multicolumn{1}{c}{\text { rotalids }} \\
\text { uvigerinids } \\
\text { Massilina } \\
\text { Sporillina } \\
\text { Elphidium }
\end{gathered}
$$

\section{MASHKID UNIT (Late Oligocene P21/P22)}

Distal-transitional facies: Inner neritic (could be a Fore-reef if larger benthic foraminifera were drifted in)

Planktonic foraminifera

Globigerina prasaepis

Globigerina cf. tripartita

Globoratalia opima nana

Catapsydrax sp. 
$\underline{\text { Benthics }}$

Nummulites sp. ex. Interc. Fabianii-fichteli

Uvigerina

Rotalia

Ditrupa

\section{ANGOHRAN UNIT: (Early Miocene with reworked Late Oligocene)}

Inner neritic or, fore-reef environment if larger benthic foraminifera were drifted in.

Planktonic foraminifera

Globigerina cf. tripartita

Globigerina prasaepis

Globigerina venezuelana

Globigerina bulloides

Globigerina ciperoensis

Globigerina aff. eupertura

Globigerina opima nana

Globigerinoides trilobus

Globoquadrina cf. dehiscens

Globigerinoides cf. sacculifer

Globigerina sellii

Globigerina aff. increbescens

Globoratalia siakensis

Catapsydrax spp.

$\underline{\text { Benthics }}$

Miogypsina thecidaeformis

Miogypsina spp.

Archaias operculiformis

Miogypsinoides spp.

Lepidocyclina $s p$.

$\underline{\text { RIG UNIT (middle to late Eocene) }}$

Planktonic foraminifera

Globigerina opima nana

Globigerina aragonensis

Globigerina angioporoides

Globigerina frantosa

Hantkenina aragonensis

JARUT UNIT (Early Miocene)

Outer neritic (Cyclammina is usually a bathyal form, but the assemblages point to an outer neritic environment) 
Planktonic foraminifera

Globigerina venezuelana

Globigerinoides spp.

Catapsydrax spp

Globigerina prasaepis

Globigerinoides trilobus

Globigerina angustiumbilicata

Lagena

Uvigerina

Benthics

Quinqueloculina

Rotalids

Polymorphinids

Bolivinids

Cyclammina

SUMMARY OF AGE CONTROL

Combining the various age constraints individual samples have uncertainties on deposition age of $\leq \pm 4$ Myrs in the Katawaz basin and $\leq \pm 5$ Myrs in the Makran.

\section{Regional U-Pb data}

Data sources for the plots used in Figure 5 are from;

DeCelles, P.G., Gehrels, G.E., Quade, J., LaReau, B. and Spurlin, M., 2000, Tectonic implications of U-Pb zircon ages of the Himalayan orogenic belt in Nepal: Science, v.288, p.497-499.

DeCelles, P.G., Gehrels, G.E., Najman, Y., Martin, A.J., Carter, A. and Garzanti, E., 2004, Detrital geochronology and geochemistry of Cretaceous-Early Miocene strata of Nepal: Implications for timing and diachroneity of initial Himalayan orogenesis: Earth and Planetary Science Letters v.227, p.313-330.

DiPietro, J. A., and Isachsen, C.E., 2001, U-Pb zircon ages from the Indian plate in northwest Pakistan and their significance to Himalayan and pre-Himalayan geologic history: Tectonics, v.20, p.510-525.

Heuberger S., Schaltegger, U., Burg, J-P., Villa, I.M., Frank, M., Dawood, H., Hussain, S., and Zanchi, A., 2007, Age and isotopic constraints on magmatism along the Karakoram- Kohistan Suture Zone, NW Pakistan: evidence for subduction and continued convergence after India-Asia collision: Swiss Journal of Geosciences, v. 100, p. $85-107$.

Khan, S.H., Walker, D.J., Hall, S.A., Burke, K.C., Shah, M.T., and Stockli, L., 2009, Did the Kohistan-Ladakh island arc collide first with India? : GSA Bulletin, v.121, p.366384.

Schaltegger, U., Zeilinger, G., Frank, M. and Burg, J.-P., 2002, Multiple mantle sources during island arc magmatism: $\mathrm{U}-\mathrm{Pb}$ and $\mathrm{Hf}$ isotopic evidence from the Kohistan arc complex, Pakistan: Terra Nova v.14, p.461-468. 
Weinberg, R.F. and Dunlap, W.J., 2000, Growth and deformation of the Ladakh batholith, northwest Himalayas: Implications for timing of continental collision and origin of calc-alkaline batholiths: Journal of Geology, v.108, p.303-320. 

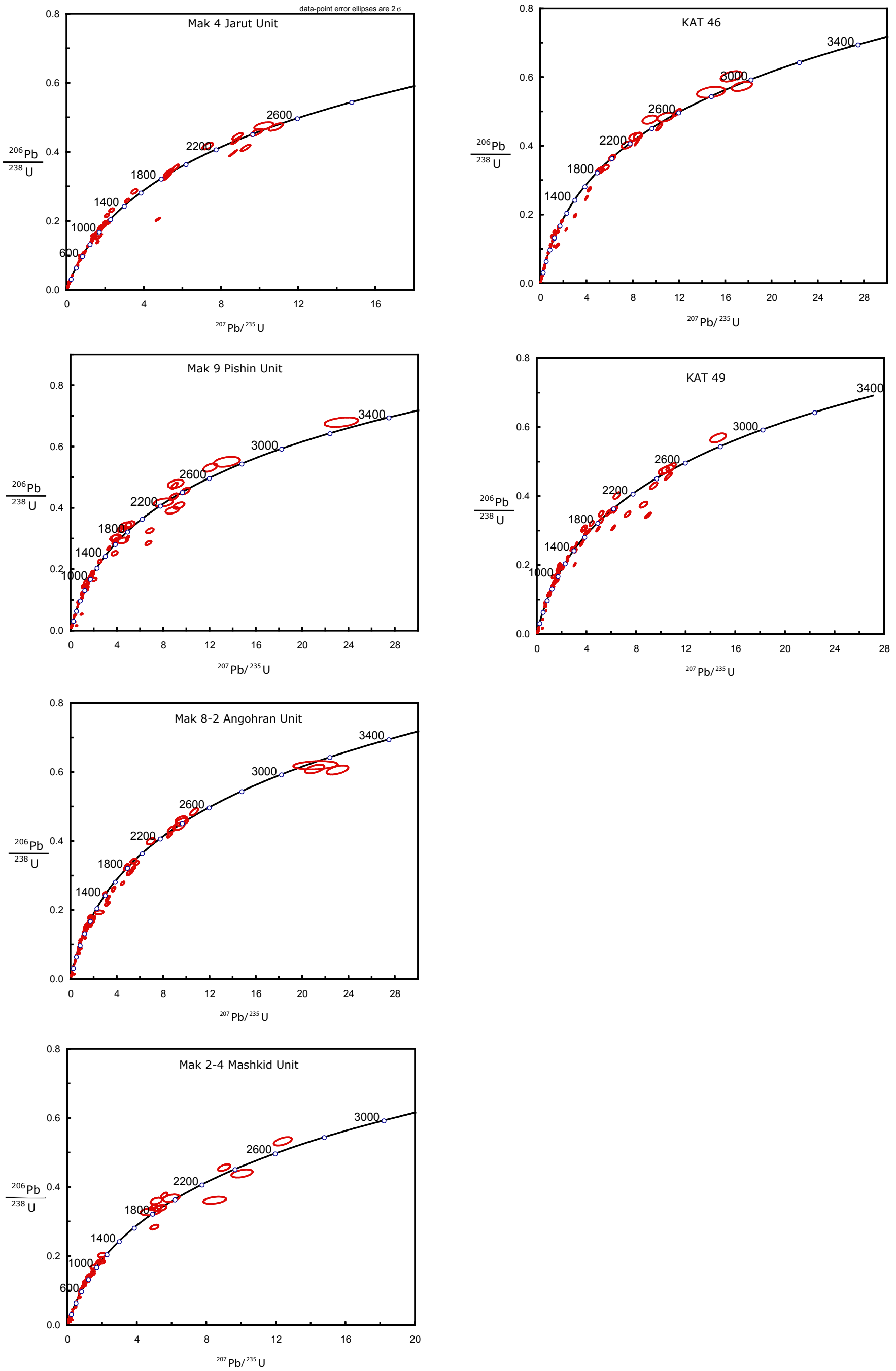


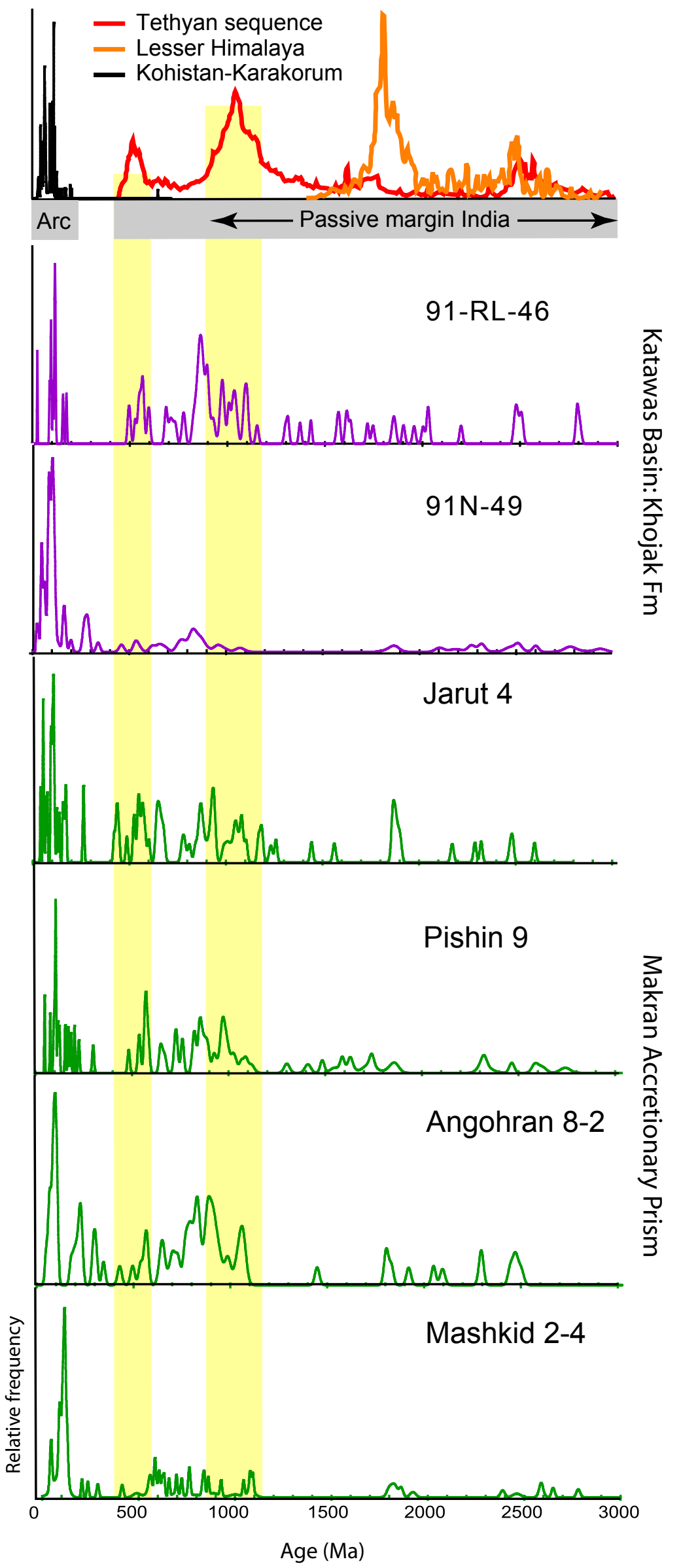


KATAWAZ BASIN

\section{Radial plots of fission track data}
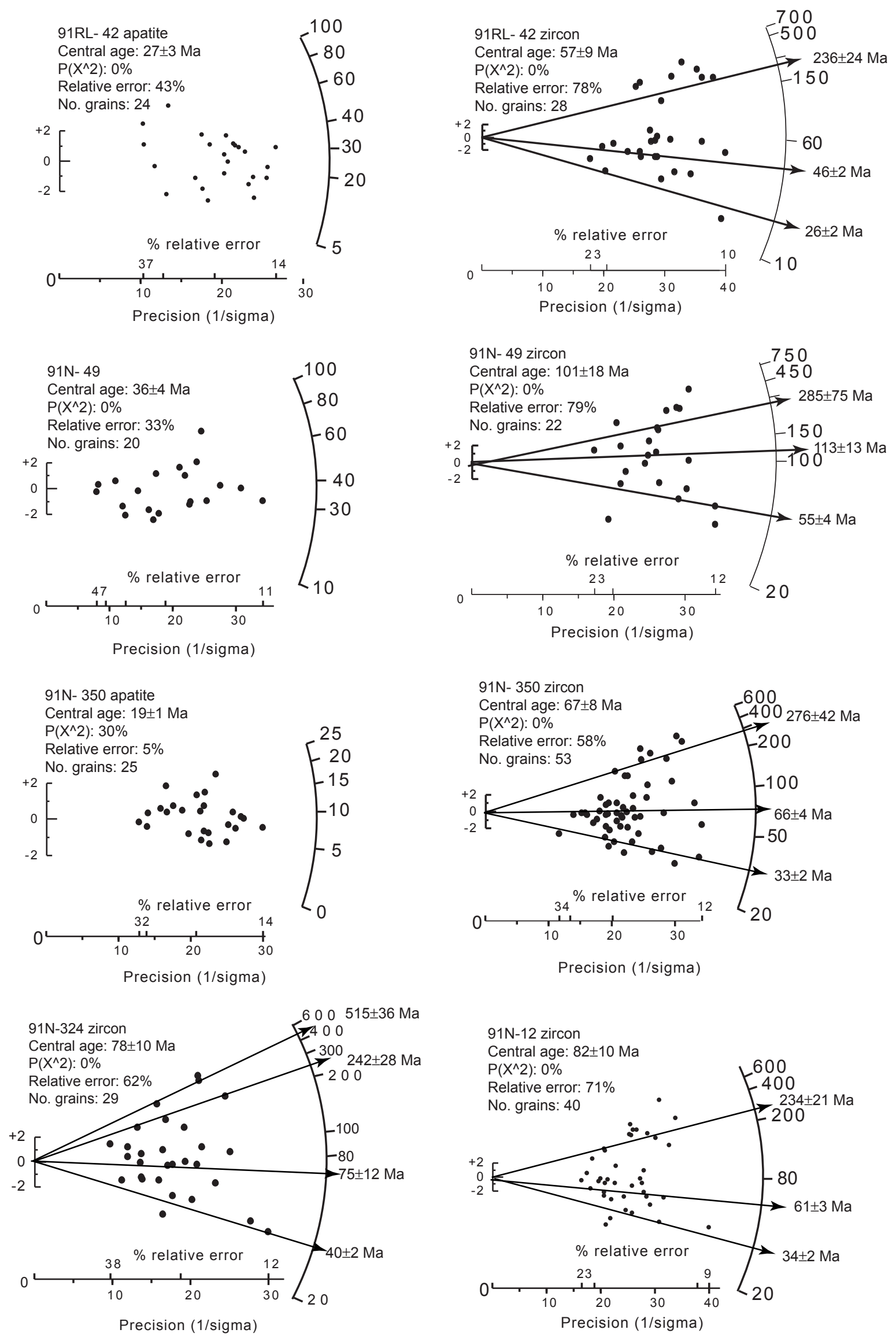


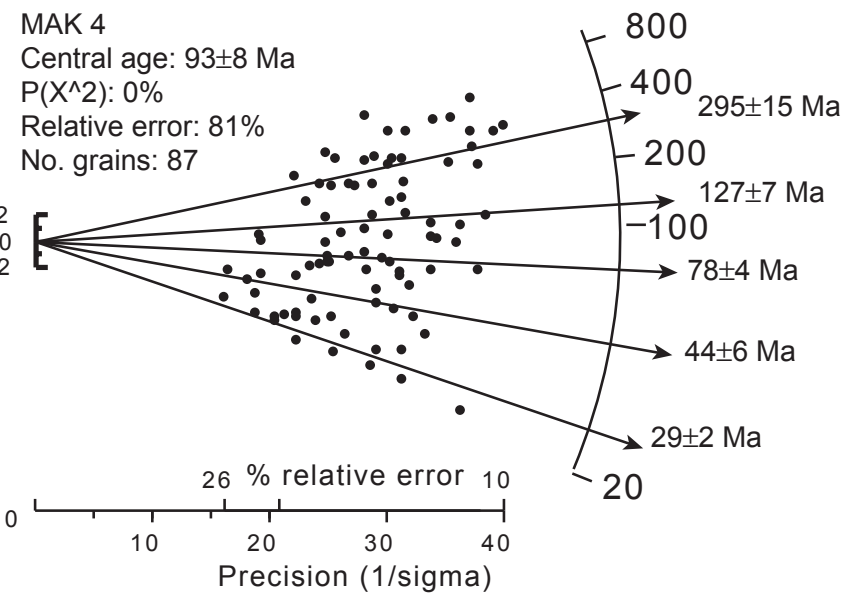

Radial plots show the individual age components for each sample derived from mixture modeling

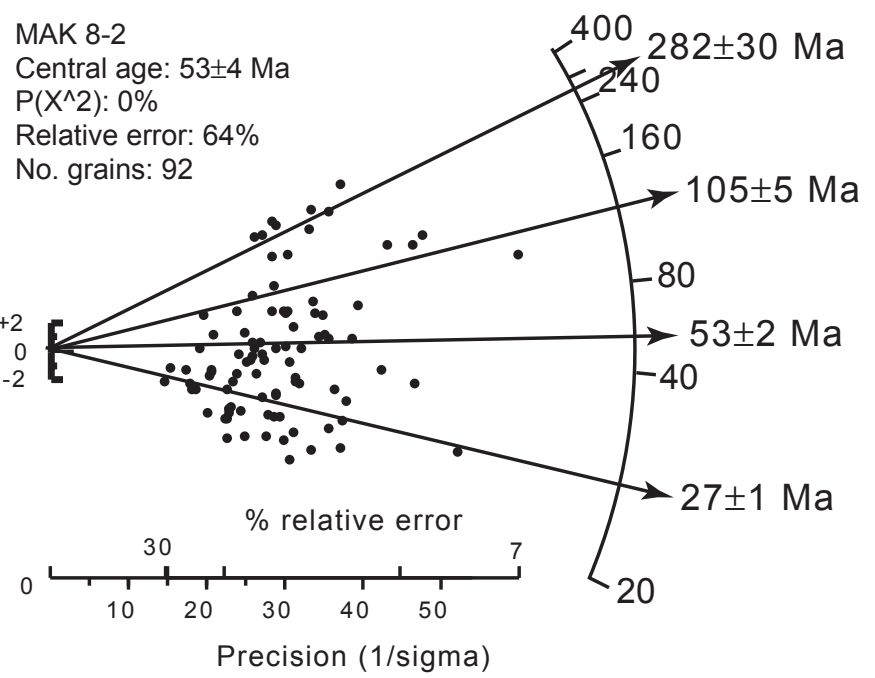

MAK 2-4

Central age: $107 \pm 7 \mathrm{Ma}$

$P\left(X^{\wedge} 2\right): 0 \%$

Relative error: $48 \%$

No. grains: 60
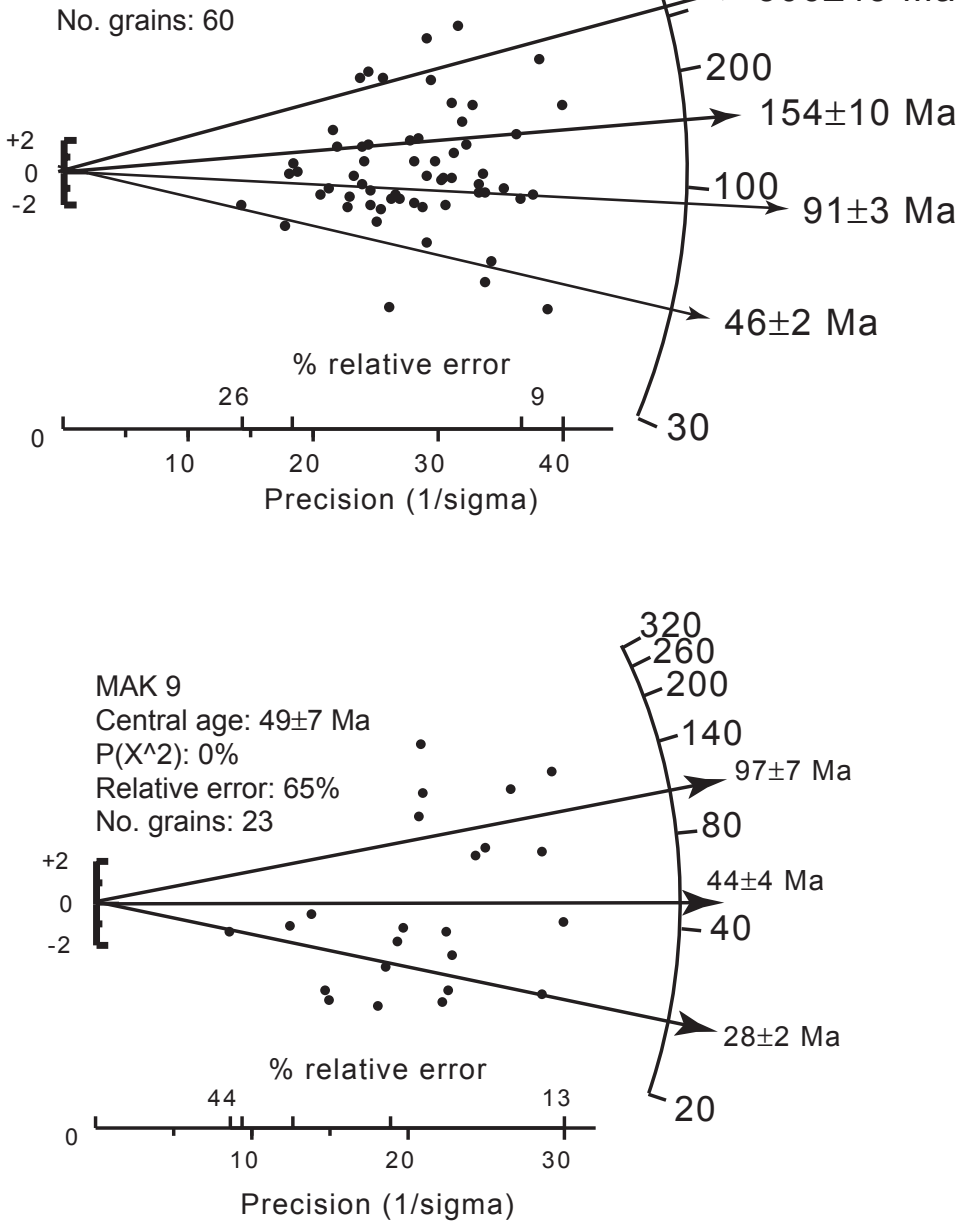


\begin{tabular}{|c|c|c|c|}
\hline MAKRAN & Grid reference & & Unit \\
\hline Sample no & Lat & Long & \\
\hline MAK 6 & N $27-24.361$ & Е $062-07.887$ & Saravan Unit \\
\hline MAK 5-1 & N $26-20.214$ & Е $060-11.897$ & Rig Unit \\
\hline MAK 1-2 & N $26-49.743$ & Е $062-38.654$ & Bamposht Unit \\
\hline MAK 2-4 & N 26 - 59.925 & E $062-31.847$ & Mashkid Unit \\
\hline MAK 8-1 & N $26-15.859$ & E $060-17.131$ & Angohran Unit \\
\hline MAK 8-2 & N $26-15.339$ & Е $060-26.709$ & Angohran Unit \\
\hline MAK 9 & N $26-04.454$ & Е $061-46.535$ & Pishin Unit \\
\hline MAK 4 & N $26-24.984$ & E $061-38.120$ & Jarut Unit \\
\hline MAK 5-2 & N $26-17.777$ & Е $060-21.585$ & Angohran Unit \\
\hline MAK 1 & N $26-26.927$ & Е $060-06.510$ & Hichan Unit (flysch) \\
\hline MAK 3 & N $26-40.531$ & Е $061-40.573$ & Nargakan Unit \\
\hline MAK 7 & N $26-54.767$ & Е $061-36.568$ & Shirinzad Unit \\
\hline MAK 2-2 & N $27-07.111$ & E $062-37.142$ & Mashkid Unit \\
\hline KATAWAZ & Grid reference & & \\
\hline Sample no & Lat & Long & Section \\
\hline $91 \mathrm{~N} 9$ & not available & & Manzaki Kili 1 \\
\hline 91N 38 & not available & & Manzaki Kili 1 \\
\hline $91 \mathrm{~N}-49$ & not available & & Manzaki Kili 1 \\
\hline 91N-98 & N 30-89.1 & Е 068-42.88 & Gardab Manda \\
\hline 91N 104 & N 30-89.27 & E 068-42.73 & Gardab Manda \\
\hline $91 \mathrm{~N} 137$ & not available & & Gardab Manda \\
\hline 91N 164 & not available & & Gardab Manda \\
\hline 91N 316 & not available & & Rud Faqirzai \\
\hline 91N 324 & not available & & Rud Faqirzai \\
\hline 91N 349 & not available & & Rud Faqirzai \\
\hline $91 \mathrm{~N}-350$ & not available & & Rud Faqirzai \\
\hline 91N 431 & N 30-93.87 & Е 068-08.58 & Nisai Railroad \\
\hline 91RL-12 & N 31-40.03 & Е 068-95.92 & Manzaki Kili 1 \\
\hline 91RL 35 & N 31-38.53 & Е 068-97.62 & Manzakai Kili 2 \\
\hline 91RL-42 & N 31-40.05 & Е 069-00.57 & Manzakai Kili 2 \\
\hline 91RL-46 & not available & & Manzaki Kili 2 \\
\hline 91MZ 27 & N 30-96.78 & Е 067-99.4 & Rud Faqirzai \\
\hline 91MZ 50 & not available & & Rud Faqirzai \\
\hline 91MZ 98 & not available & & Nisai Railroad \\
\hline
\end{tabular}




\begin{tabular}{|c|c|c|c|c|c|c|c|c|c|c|c|c|c|c|c|c|c|}
\hline Formation & section & Sample & Mineral & $\begin{array}{l}\text { Number } \\
\text { of Grains }\end{array}$ & Pd & $\mathbf{N}_{\mathrm{d}}$ & Ps & Ns & $\mathbf{P i}$ & $\mathrm{Ni}$ & Chi 2 & RE\% & $\begin{array}{c}\text { Central } \\
\text { Age } \\
\text { (Ma) }\end{array}$ & $\mathrm{P} 1$ & $\begin{array}{l}\text { Age components } \\
\text { P2 }\end{array}$ & P3 & $\mathrm{P} 4$ \\
\hline \multicolumn{18}{|l|}{ KATAWAZ } \\
\hline $\begin{array}{l}\text { Shaigalu } \\
\text { Shaigalu }\end{array}$ & Manzaki Killi & $91 \mathrm{~N}-49 \mathrm{z}$ & Zircon & 22 & 0.364 & 1200 & $\begin{array}{l}0.422 \\
11.85\end{array}$ & 3202 & 2.768 & 748 & 0 & $\begin{array}{l}37.8 \\
79.3\end{array}$ & $100 \pm 17$ & $\begin{array}{l}18 \pm 5(3) \\
29 \pm 3(1)\end{array}$ & $\begin{array}{l}34 \pm 4 \text { (12) } \\
55 \pm 4(7)\end{array}$ & $\begin{array}{l}62 \pm 10(5) \\
113 \pm 13(8)\end{array}$ & $285 \pm 75(7)$ \\
\hline Nisai & Gardab Manda & $91 \mathrm{~N}-98$ & Apatite & 31 & 1.299 & 7200 & 0.021 & 228 & 0.059 & 650 & 0 & 42.9 & $80 \pm 9$ & $59 \pm 6(28)$ & 200-300 (3) & & \\
\hline Shaigalu & Rud Faqirzai & $91 \mathrm{~N}-350$ & Apatite & 25 & 1.299 & 7200 & 0.013 & 115 & 3.091 & 2609 & 30 & 15.5 & $10 \pm 1$ & Single population & & & \\
\hline Shaigalu & Rud Faqirzai & $91 \mathrm{~N}-350 \mathrm{z}$ & Zircon & 53 & 0.366 & 2550 & 11 & 5319 & 3.612 & 1747 & 0 & 58.3 & $67 \pm 6$ & $33 \pm 2(18)$ & $66 \pm 4(30)$ & $276 \pm 42(5)$ & \\
\hline Shaigalu & Manzaki & 91RL-12 & Apatite & 31 & 1.299 & 7200 & 0.031 & 344 & 3.672 & 4041 & 9 & 16.8 & $19 \pm 1$ & Single population & & & \\
\hline Shaigalu & Manzaki & 91RL-12z & Zircon & 40 & 0.369 & 2550 & 12.12 & 5729 & 3.371 & 1593 & 0 & 70.6 & $82 \pm 10$ & $34 \pm 2(8)$ & $61 \pm 3(20)$ & $234 \pm 21(12)$ & \\
\hline Shaigalu & Manzaki & 91RL-42 & Apatite & 25 & 1.299 & 7200 & 0.262 & 276 & 2.231 & 2350 & 0 & 43.2 & $27 \pm 3$ & $11 \pm 3(6)$ & $20 \pm 6(8)$ & $30 \pm 6(10)$ & \\
\hline Shaigalu & Manzaki & 91RL-42z & Zircon & 28 & 0.371 & 2550 & 8.863 & 4275 & 3.479 & 1678 & 0 & 77.6 & $57 \pm 9$ & $16 \pm 2(1)$ & $27 \pm 2(6)$ & $46 \pm 2(14)$ & $236 \pm 24(7)$ \\
\hline Shaigalu & Manzaki 2 & 91RL-46 & Apatite & 25 & 1.299 & 7200 & 0.261 & 280 & 1.865 & 2001 & 0 & 15.4 & $31 \pm 2$ & $22 \pm 5(15)$ & $33 \pm 5(8)$ & 200-300 (2) & \\
\hline MF & Rud Faqirzai & 91N324 & Zircon & 29 & 0.346 & 2437 & 9.329 & 2211 & 2.776 & 658 & 0 & 62.2 & $78 \pm 10$ & $75 \pm 4(12)$ & $40 \pm 2(11)$ & $242 \pm 28(3)$ & $515 \pm 36(2)$ \\
\hline \multicolumn{18}{|l|}{ MAKRAN } \\
\hline Saravan Unit & & MAK 6 & Zircon & 30 & 0.625 & 3433 & 15.54 & 5560 & 5.386 & 2063 & 0 & 50.1 & $164 \pm 10$ & & & & \\
\hline Saravan Unit & & MAK 6 & Apatite & 9 & 1.407 & 5879 & 0.342 & 99 & 2.406 & 657 & 2.2 & 33.3 & $37 \pm 6$ & Single population & & & \\
\hline Rig Unit & & MAK 5-1 & Zircon & 54 & 0.625 & 3433 & 8.648 & 4719 & 3.36 & 1883 & 0 & 32 & $93 \pm 5$ & $73 \pm 3(32)$ & $113 \pm 8(21)$ & & \\
\hline Rig Unit & & MAK 5-1 & Apatite & 36 & 1.407 & 5879 & 0.362 & 374 & 4.82 & 5213 & 32.2 & 11.8 & $17 \pm 1$ & Single population & & & \\
\hline Bamposht Unit & & MAK 1-2 & Zircon & 19 & 0.625 & 3433 & 13.28 & 2538 & 4.532 & 845 & 0 & 37.3 & $120 \pm 12$ & $40 \pm 6(1)$ & $128 \pm 18(18)$ & & \\
\hline Mashkid Unit & & MAK 2-4 & Zircon & 60 & 0.625 & 3433 & 15.92 & 11264 & 4.909 & 3428 & 0 & 48.3 & $107 \pm 7$ & $46 \pm 2(7)$ & $92 \pm 3(36)$ & $165 \pm 11(16)$ & \\
\hline Angohran Unit & & MAK 8-1 & Zircon & 52 & 0.625 & 3433 & 7.747 & 4880 & 4.963 & 3140 & 0 & 60.9 & $46 \pm 4$ & $23 \pm 1(18)$ & $39.8 \pm 2.9(17)$ & $80 \pm 9(12)$ & $307 \pm 39(4)$ \\
\hline Angohran Unit & & MAK 8-1 & Apatite & 35 & 1.407 & 5879 & 0.269 & 453 & 3.912 & 6650 & 31.61 & 10.2 & $16 \pm 1$ & Single population & & & \\
\hline Angohran Unit & & MAK 8-2 & Zircon & 40 & 0.625 & 3433 & 8.303 & 12345 & 5.049 & 7749 & 0 & 62.4 & $62 \pm 4$ & & & & \\
\hline Pishin Unit & & MAK 9 & Zircon & 23 & 0.625 & 3433 & 8.312 & 1933 & 4.775 & 1152 & 0 & 65.3 & $49 \pm 7$ & & & & \\
\hline Pishin Unit & & MAK 9 & Apatite & 20 & 1.407 & 5879 & 0.289 & 196 & 5.403 & 4179 & 0.22 & 34.7 & $11 \pm 1$ & Single population & & & \\
\hline Jarut Unit & & MAK 4 & Zircon & 87 & 0.625 & 3433 & 13.93 & 12199 & 4.793 & 4463 & 0 & 81 & $93 \pm 8$ & $29 \pm 2(14)$ & $44 \pm 6(11)$ & $78 \pm 4(19)$ & $127 \pm 4(19$ \\
\hline
\end{tabular}

\section{Table 1: Fission track analytical data}

(i). Track densities are (x106 tr cm-2) numbers of tracks counted (N) shown in brackets;

(ii). analyses by external detector method using 0.5 for the $4 \square / 2 \square$ geometry correction factor;

(iii). ages calculated using dosimeter glass CN-5; (apatite) $\square \mathrm{CN} 5=338 \pm 4$; CN-2 (zircon) $\square \mathrm{CN} 2=127 \pm 4$

calibrated by multiple analyses of IUGS apatite and zircon age standards (see Hurford 1990);

(iv). $P \sqsubset 2$ is probability for obtaining $\square 2$ value for $v$ degrees of freedom, where $v=$ no. crystals -1 ;

(v). Central age is a modal age, weighted for different precisions of individual crystals (see Galbraith 1992);

(vi) Age modes deconvolved using approach of Sambridge \& Compston (1994) and Galbraith \& Green (1983)

\begin{tabular}{|l|l|}
\hline \multicolumn{2}{|c|}{ Fission Track (all data) } \\
\hline \multicolumn{1}{|c|}{ Makran } & Katawaz \\
\hline $28 \pm 1(42)$ & $31 \pm 1(34)$ \\
\hline $52 \pm 2(54)$ & $57 \pm 2(65)$ \\
\hline $99 \pm 2(93)$ & $113 \pm 9(16)$ \\
\hline $276 \pm 13(42)$ & $270 \pm 30(34)$ \\
\hline & \\
\hline
\end{tabular}

Table 2 Summary of mixture modeling 
Apparent Ages (Ma)

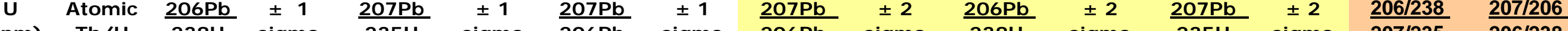

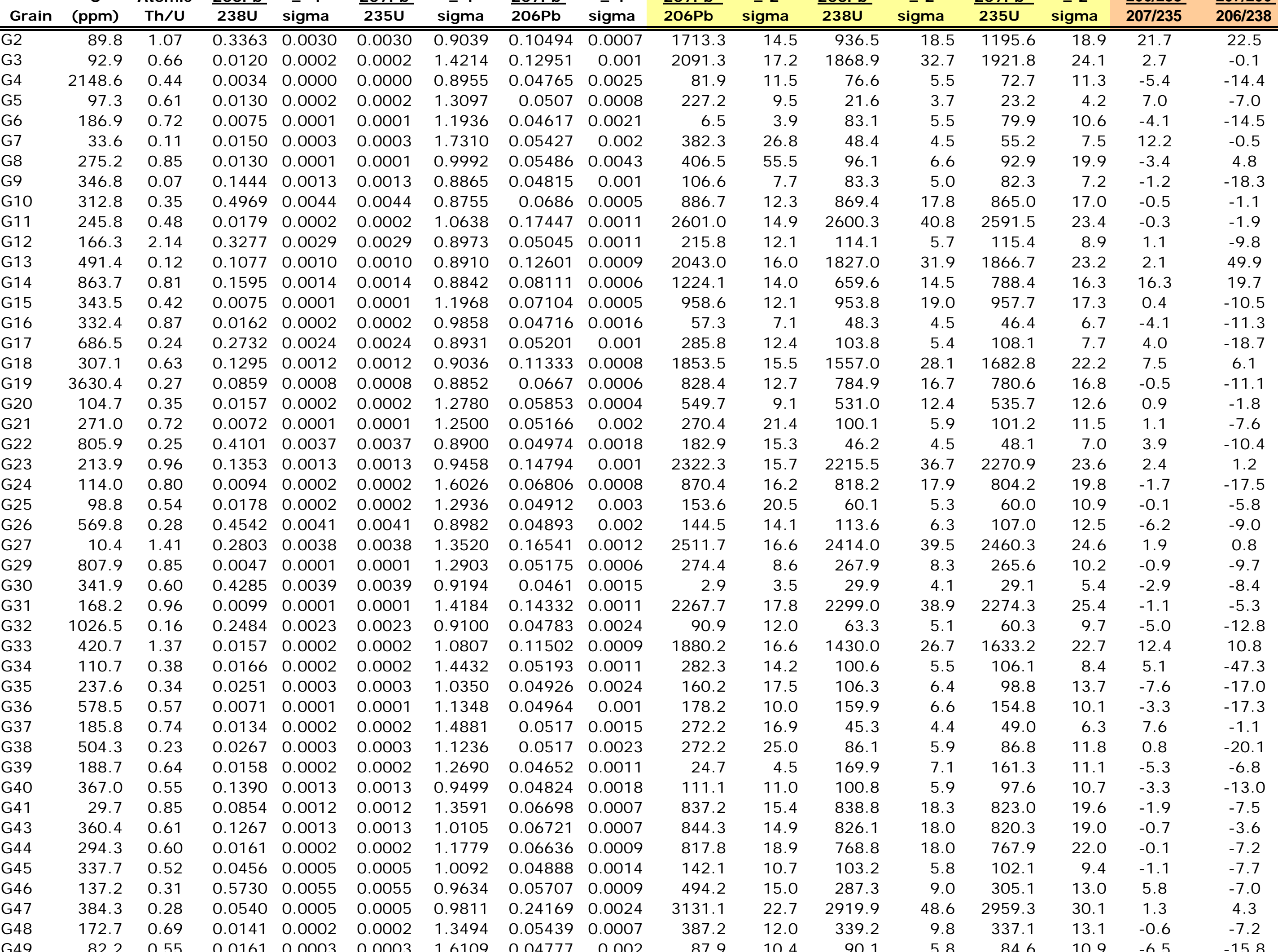


$\begin{array}{llllllll}271.1 & 0.25 & 0.3655 & 0.0035 & 0.0035 & 0.9658 & 0.04815 & 0.003\end{array}$

$\begin{array}{lllllllllll}1925.6 & 0.15 & 0.1964 & 0.0019 & 0.0019 & 0.9571 & 0.13043 & 0.0014 & 106.6\end{array}$

$\begin{array}{lllllllll}516.5 & 0.31 & 0.0313 & 0.0004 & 0.0004 & 1.1179 & 0.10983 & 0.0012\end{array}$

$\begin{array}{lllllllll}470.7 & 0.35 & 0.0259 & 0.0003 & 0.0003 & 1.0433 & 0.05165 & 0.0012\end{array}$

$\begin{array}{lllllllll}522.6 & 0.47 & 0.0452 & 0.0005 & 0.0005 & 1.0175 & 0.05014 & 0.0009 \\ 631.9 & 0.11 & 0.3292 & 0.0032 & 0.0032 & 0.9689 & 0.05217 & 0.0008\end{array}$

$\begin{array}{lllllllll}443.4 & 0.48 & 0.0173 & 0.0002 & 0.0002 & 1.1561 & 0.11459 & 0.0013\end{array}$

$\begin{array}{lllllllll}70.2 & 0.36 & 0.6028 & 0.0062 & 0.0062 & 1.0202 & 0.04831 & 0.0012\end{array}$

$\begin{array}{lllllllll}923.1 & 0.16 & 0.1066 & 0.0010 & 0.0010 & 0.9754 & 0.19407 & 0.0024\end{array}$

$\begin{array}{lllllllll}614.0 & 0.85 & 0.0130 & 0.0001 & 0.0001 & 1.0769 & 0.06148 & 0.0007\end{array}$

$\begin{array}{lllllllll}343.1 & 0.72 & 0.1810 & 0.0018 & 0.0018 & 0.9892 & 0.04772 & 0.001\end{array}$

$\begin{array}{lllllllll}330.4 & 0.58 & 0.0459 & 0.0005 & 0.0005 & 1.0685 & 0.07592 & 0.001\end{array}$

$\begin{array}{llllllll}172.5 & 0.70 & 0.0083 & 0.0001 & 0.0001 & 1.5625 & 0.06295 & 0.0011\end{array}$

$\begin{array}{lllllllll}123.9 & 0.53 & 0.0169 & 0.0003 & 0.0003 & 1.4828 & 0.04718 & 0.0029\end{array}$

$\begin{array}{rrrrrrrr}342.5 & 1.28 & 0.0132 & 0.0002 & 0.0002 & 1.2094 & 0.0494 & 0.0023 \\ 102.0 & 0.80 & 0.4761 & 0.0049 & 0.0049 & 1.0292 & 0.04872 & 0.0015\end{array}$

$\begin{array}{llllllll}336.7 & 0.53 & 0.1242 & 0.0013 & 0.0013 & 1.0148 & 0.16113 & 0.0022\end{array}$

$\begin{array}{lllllllll}217.0 & 0.51 & 0.0127 & 0.0002 & 0.0002 & 1.4140 & 0.06544 & 0.0009\end{array}$

$\begin{array}{llllllll}322.4 & 1.93 & 0.0434 & 0.0005 & 0.0005 & 1.0832 & 0.0524 & 0.0022\end{array}$

$\begin{array}{lllllllll}316.7 & 0.11 & 0.1008 & 0.0010 & 0.0010 & 1.0223 & 0.05651 & 0.0011\end{array}$

$\begin{array}{lllllllll}138.3 & 0.32 & 0.6021 & 0.0061 & 0.0061 & 1.0149 & 0.06062 & 0.0009\end{array}$

$\begin{array}{lllllllll}173.5 & 0.97 & 0.0146 & 0.0002 & 0.0002 & 1.3736 & 0.21383 & 0.0029\end{array}$

$\begin{array}{lllllllll}444.7 & 1.07 & 0.0073 & 0.0001 & 0.0001 & 1.3755 & 0.04776 & 0.0019\end{array}$

$\begin{array}{rrrrrrrrr}354.3 & 0.26 & 0.0740 & 0.0008 & 0.0008 & 1.0680 & 0.04696 & 0.0018\end{array}$

$\begin{array}{rllllllll}1029.5 & 0.79 & 0.0073 & 0.0001 & 0.0001 & 1.0944 & 0.05924 & 0.001\end{array}$

$\begin{array}{lllllllll}126.2 & 2.89 & 0.1490 & 0.0017 & 0.0017 & 1.1681 & 0.04697 & 0.0011\end{array}$

$\begin{array}{lllllllll}297.0 & 0.64 & 0.0453 & 0.0005 & 0.0005 & 1.1030 & 0.07207 & 0.0048\end{array}$

$\begin{array}{lllllllll}436.2 & 0.67 & 0.1367 & 0.0014 & 0.0014 & 1.0385 & 0.05269 & 0.0011\end{array}$

$\begin{array}{lllllllll}261.6 & 0.57 & 0.0137 & 0.0002 & 0.0002 & 1.3909 & 0.06758 & 0.0011\end{array}$

$\begin{array}{llllllll}86.9 & 2.55 & 0.1358 & 0.0017 & 0.0017 & 1.2150 & 0.06948 & 0.0012\end{array}$

$\begin{array}{llllllll}427.1 & 0.60 & 0.4023 & 0.0042 & 0.0042 & 1.0540 & 0.0651 & 0.0016\end{array}$

$\begin{array}{llllllll}727.6 & 0.23 & 0.0253 & 0.0003 & 0.0003 & 1.1444 & 0.13684 & 0.0023\end{array}$

$\begin{array}{rrrrrrrrr}1380.1 & 0.25 & 0.0160 & 0.0002 & 0.0002 & 1.1264 & 0.04987 & 0.0011 \\ 93.5 & 0.44 & 0.0171 & 0.0003 & 0.0003 & 1.7503 & 0.0483 & 0.001\end{array}$

$\begin{array}{rrrrrrrr}93.5 & 0.44 & 0.0171 & 0.0003 & 0.0003 & 1.7503 & 0.0483 & 0.001 \\ 719.4 & 0.57 & 0.3277 & 0.0035 & 0.0035 & 1.0680 & 0.05464 & 0.0033\end{array}$

$\begin{array}{llllllll}468.6 & 0.66 & 0.0134 & 0.0002 & 0.0002 & 1.3453 & 0.11311 & 0.002\end{array}$

$\begin{array}{llllllll}204.9 & 0.49 & 0.4265 & 0.0046 & 0.0046 & 1.0832 & 0.05019 & 0.0017\end{array}$

$\begin{array}{llllllll}434.3 & 0.39 & 0.4830 & 0.0052 & 0.0052 & 1.0786 & 0.1466 & 0.0027\end{array}$

$\begin{array}{lllllllll}104.0 & 0.49 & 0.0170 & 0.0003 & 0.0003 & 1.7689 & 0.16418 & 0.003\end{array}$

$\begin{array}{lllllllll}2044.9 & 0.05 & 0.1124 & 0.0012 & 0.0012 & 1.0858 & 0.06166 & 0.0033\end{array}$

$\begin{array}{lllllllll}113.9 & 1.75 & 0.1499 & 0.0018 & 0.0018 & 1.1678 & 0.09887 & 0.0019\end{array}$

$\begin{array}{lllllllll}326.3 & 0.57 & 0.0101 & 0.0002 & 0.0002 & 1.4866 & 0.06901 & 0.0015\end{array}$

$\begin{array}{llllllll}317.2 & 0.88 & 0.0094 & 0.0002 & 0.0002 & 1.6009 & 0.0472 & 0.0022\end{array}$

$\begin{array}{rrrrrrrr}552.1 & 0.81 & 0.0210 & 0.0003 & 0.0003 & 1.1882 & 0.0494 & 0.0025 \\ 2736.0 & 0.52 & 0.0070 & 0.0001 & 0.0001 & 1.2950 & 0.06208 & 0.0015\end{array}$

$\begin{array}{rrrrrrrrr}2736.0 & 0.52 & 0.0070 & 0.0001 & 0.0001 & 1.2950 & 0.06208 & 0.0015 \\ 207.9 & 0.35 & 0.1394 & 0.0016 & 0.0016 & 1.1548 & 0.0579 & 0.0017\end{array}$

$\begin{array}{rrrrrrrr}207.9 & 0.35 & 0.1394 & 0.0016 & 0.0016 & 1.1548 & 0.0579 & 0.0017 \\ 25.8 & 0.65 & 0.0207 & 0.0006 & 0.0006 & 2.7536 & 0.06763 & 0.0015\end{array}$

$\begin{array}{llllllllll}929.2 & 0.75 & 0.0175 & 0.0002 & 0.0002 & 1.2007 & 0.07906 & 0.0077\end{array}$

$\begin{array}{llllllll}1.5 & 1.73 & 0.7106 & 0.0496 & 0.0496 & 6.9853 & 0.04858 & 0.0012\end{array}$

$\begin{array}{lllllllll}193.8 & 0.56 & 0.0156 & 0.0002 & 0.0002 & 1.5365 & 0.0623 & 0.0014\end{array}$

$\begin{array}{llllllll}407.6 & 0.39 & 0.1647 & 0.0019 & 0.0019 & 1.1657 & 0.05895 & 0.0014\end{array}$

$\begin{array}{lllllllll}226.7 & 1.70 & 0.0184 & 0.0003 & 0.0003 & 1.5226 & 0.07216 & 0.0017\end{array}$

$\begin{array}{llllllllll}\text { G109 } & 1370.7 & 2.14 & 0.0417 & 0.0005 & 0.0005 & 1.1762 & 0.04831 & 0.0021\end{array}$

$\begin{array}{llllllllll}\text { G110 } & 412.9 & 0.54 & 0.5558 & 0.0065 & 0.0065 & 1.1659 & 0.05324 & 0.0013\end{array}$

$22.4 \quad 2008.1$

$21.0 \quad 1156.1$

$14.1 \quad 198.7$

$10.0 \quad 164.7$

$\begin{array}{ll}11.3 & 285.0\end{array}$

$1873.4 \quad 22.4 \quad 1834.7$

$\begin{array}{rrr}114.5 & 8.9 & 110.6\end{array}$

$\begin{array}{rrr}656.1 & 15.0 & 653.1\end{array}$

$\begin{array}{lll}85.4 & 6.8 & 83.3\end{array}$

$\begin{array}{lll}1093.0 & 20.3 & 1072.2\end{array}$

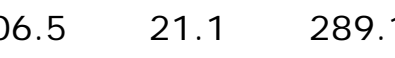

$\begin{array}{rrr}58.3 & 10.2 & 53.4 \\ 166.9 & 17.4 & 107.8\end{array}$

$\begin{array}{rrr}134.4 & 10.8 & 84.7\end{array}$

2467.6

788.5

302.9
472.5

625.8

\begin{tabular}{ll}
17.4 & 273.8 \\
\hline & 618.8
\end{tabular}

$\begin{array}{rrr}87.4 & 10.1 & 93.2 \\ 47.2 & 6.9 & 46.7\end{array}$

$\begin{array}{rrr}87.4 & 10.1 & 93.2 \\ 575.9 & 18.6 & 460.7\end{array}$

$5.6 \quad 47.0$

$\begin{array}{lll}1270.6 & 32.7 & 895.1\end{array}$

$\begin{array}{lll}987.9 & 88.2 & 21.8\end{array}$

$\begin{array}{rr}88.2 & 21.8 \\ 14.3 & 285.8\end{array}$

$\begin{array}{ll}14.3 & 285.8 \\ 21.8 & 826.2\end{array}$

$\begin{array}{ll}21.8 & 826.2 \\ 24.1 & 868.9\end{array}$

$\begin{array}{ll}29.4 & 820.9\end{array}$

$33.4 \quad 2179.5$

$11.1 \quad 161.3$

$\begin{array}{rr}7.9 & 102.2\end{array}$

$\begin{array}{lr}42.5 & 109.6\end{array}$

33.31827 .3

$\begin{array}{lr}15.5 & 85.7 \\ 36.4 & 2290.0\end{array}$

$\begin{array}{ll}36.4 & 2290.0\end{array}$

$\begin{array}{rr}37.3 & 2540.4 \\ 55.6 & 108.4\end{array}$

$\begin{array}{ll}55.6 & 108.4 \\ 33.7 & 686.4\end{array}$

$\begin{array}{ll}33.7 & 686.4 \\ 29.8 & 900.2\end{array}$

$\begin{array}{rr}29.8 & 900.2 \\ 8.6 & 64.7\end{array}$

$\begin{array}{rr}8.6 & 64.7 \\ 18.6 & 60.1\end{array}$

$26.9 \quad 134.2$

$26.7 \quad 44.6$

$29.0 \quad 841.4$

$138.0 \quad 132.1$

$9.3 \quad 111.8$

$\begin{array}{ll}26.4 & 681.9 \\ 23.8 & 547.4\end{array}$

$32.6 \quad 982.9$

$\begin{array}{ll}32.6 & 982.9 \\ 12.8 & 117.5\end{array}$

$17.2 \quad 263.1$

$47.7 \quad 2849.2$

$\begin{array}{rrrcc}6.6 & 94.5 & 15.9 & -9.2 & -4.9 \\ 36.7 & 2008.5 & 28.8 & 0.0 & 1.0 \\ 23.6 & 1407.1 & 24.9 & 17.8 & 16.3 \\ 7.7 & 200.1 & 12.7 & 0.7 & 6.7 \\ 6.7 & 165.1 & 9.8 & 0.2 & -3.6 \\ 9.0 & 283.2 & 12.7 & -0.7 & -3.8 \\ 34.3 & 1849.3 & 28.5 & 0.8 & 0.2 \\ 5.9 & 107.7 & 9.2 & -2.7 & -3.5 \\ 52.8 & 2737.0 & 33.6 & -11.1 & -6.0 \\ 15.5 & 654.8 & 18.3 & 0.3 & -1.9 \\ 5.1 & 81.6 & 7.2 & -2.1 & -4.3 \\ 22.9 & 1070.2 & 24.7 & -0.2 & -6.4 \\ 9.4 & 338.3 & 15.2 & 14.6 & 0.5 \\ 5.0 & 50.8 & 9.8 & -5.1 & -11.3 \\ 6.5 & 102.0 & 13.4 & -5.7 & -28.9 \\ 5.4 & 83.3 & 8.7 & -1.7 & -39.9 \\ 46.1 & 2384.4 & 34.8 & -5.3 & -6.3 \\ 17.8 & 743.3 & 22.1 & -1.5 & -4.8 \\ 5.6 & 82.9 & 10.8 & 1.7 & -8.2 \\ 9.2 & 284.9 & 14.4 & 3.9 & 68.2 \\ 15.4 & 608.1 & 20.2 & -1.8 & -6.4 \\ 52.5 & 2907.9 & 36.1 & -4.5 & -2.2 \\ 5.9 & 88.2 & 10.9 & -5.6 & -10.0 \\ 4.6 & 45.4 & 7.0 & -2.9 & -16.0 \\ 12.8 & 465.4 & 18.9 & 1.2 & 4.8 \\ 4.4 & 47.1 & 5.8 & 0.3 & -2.5 \\ 22.9 & 927.9 & 33.2 & 3.5 & 83.9 \\ 4.2 & 32.1 & 8.0 & 32.2 & 13.9 \\ 9.5 & 282.9 & 14.9 & -1.0 & -6.0 \\ 19.4 & 821.5 & 25.0 & -0.6 & -3.9 \\ 20.7 & 857.8 & 27.3 & -1.3 & -3.9 \\ 22.1 & 714.8 & 33.3 & -14.8 & 74.3 \\ 42.3 & 2172.1 & 38.8 & -0.3 & -4.7 \\ 7.0 & 161.6 & 10.9 & 0.2 & -1.8 \\ 5.6 & 103.0 & 8.1 & 0.8 & -2.5 \\ 7.1 & 107.9 & 17.7 & -1.6 & -22.7 \\ 37.3 & 1844.2 & 38.3 & 0.9 & -4.1 \\ 5.6 & 87.5 & 9.5 & 2.1 & -12.4 \\ 45.1 & 2259.9 & 41.8 & -1.3 & -2.2 \\ 48.6 & 2507.9 & 42.8 & -1.3 & -3.4 \\ 7.1 & 117.2 & 17.8 & 7.5 & -44.0 \\ 17.5 & 943.5 & 30.0 & 27.2 & 20.1 \\ 23.0 & 816.7 & 33.3 & -10.2 & -158.9 \\ 5.3 & 61.3 & 9.4 & -5.6 & -8.8 \\ 5.3 & 59.7 & 9.7 & -0.6 & -7.0 \\ 6.5 & 163.7 & 11.6 & 18.0 & 2.7 \\ 4.5 & 54.9 & 6.7 & 18.7 & 3.7 \\ 21.6 & 825.3 & 32.0 & -2.0 & -7.1 \\ 10.5 & 140.9 & 41.0 & 6.3 & 10.0 \\ 6.0 & 110.0 & 9.2 & -1.7 & -1.9 \\ 18.4 & 649.4 & 29.1 & -5.0 & -11.0 \\ 15.7 & 540.9 & 25.7 & -1.2 & -6.2 \\ 24.6 & 986.3 & 36.0 & 0.3 & -4.3 \\ 6.9 & 109.6 & 13.6 & -7.2 & -670.1 \\ 9.4 & 270.7 & 16.1 & 2.8 & 119.2 \\ 57.0 & 2801.0 & 53.0 & -1.7 & -4.7\end{array}$


SAMPLE: KAT $91 \mathrm{~N}-49$

\section{Apparent Ages (Ma)}

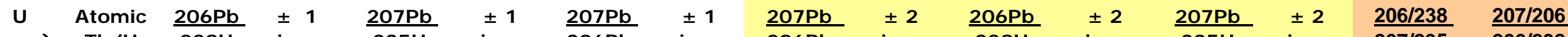

\begin{tabular}{|c|c|c|c|c|c|c|c|c|c|c|c|c|c|c|c|c|}
\hline Grain & (ppm) & $\mathrm{Th} / \mathrm{U}$ & 2380 & sigma & 2350 & sigma & $206 \mathrm{~Pb}$ & sigma & $206 \mathrm{~Pb}$ & sigma & 2380 & sigma & 2350 & sigma & $207 / 235$ & $206 / 238$ \\
\hline $\mathrm{G} 2$ & 691 & 0.3206 & 0.2599 & 0.0024 & 3.17851 & 0.0307 & 0.09011 & 0.0006 & 1427.9 & 12.0 & 1489.5 & 26.5 & 1452.0 & 19.5 & -2.6 & -4.7 \\
\hline 53 & 591 & 0.25569 & 0.1445 & 0.0013 & 1.32257 & 0.0144 & 0.06794 & 0.0005 & 866.7 & 10.9 & 869.9 & 17.1 & 855.6 & 16.0 & -1.7 & -3.9 \\
\hline G4 & 791 & 0.85418 & 0.3441 & 0.0032 & 8.96114 & 0.0942 & 0.18516 & 0.0011 & 2699.6 & 13.7 & 1906.1 & 32.3 & 2334.1 & 22.2 & 18.3 & -2.4 \\
\hline $\mathrm{G} 6$ & 1090 & 0.14819 & 0.0977 & 0.0009 & 0.79621 & 0.0075 & 0.05986 & 0.0004 & 598.5 & 8.8 & 600.6 & 12.7 & 594.7 & 12.8 & -1.0 & -2.2 \\
\hline G7 & 187 & 0.30224 & 0.5686 & 0.0053 & 14.6151 & 0.2581 & 0.19603 & 0.0013 & 2793.4 & 14.6 & 2902.1 & 45.8 & 2790.5 & 23.9 & -4.0 & -2.7 \\
\hline G8 & 92 & 0.36899 & 0.1489 & 0.0016 & 1.41105 & 0.0436 & 0.07608 & 0.0013 & 1097.2 & 24.1 & 894.7 & 20.3 & 893.6 & 26.5 & -0.1 & -6.3 \\
\hline 69 & 645 & 1.40137 & 0.1688 & 0.0016 & 1.70738 & 0.0179 & 0.0746 & 0.0005 & 1057.7 & 11.4 & 1005.2 & 19.2 & 1011.3 & 17.1 & 0.6 & -40.1 \\
\hline G13 & 368 & 0.34333 & 0.1554 & 0.0015 & 1.69314 & 0.0231 & 0.08247 & 0.0007 & 1256.7 & 14.0 & 931.3 & 18.4 & 1005.9 & 18.3 & 7.4 & -0.3 \\
\hline G14 & 263 & 0.62133 & 0.1953 & 0.0018 & 1.94732 & 0.0294 & 0.07627 & 0.0007 & 1102.2 & 13.6 & 1150.0 & 22.0 & 1097.5 & 19.5 & -4.8 & -7.7 \\
\hline G15 & 882 & 0.4739 & 0.0276 & 0.0003 & 0.18682 & 0.0029 & 0.04972 & 0.0007 & 181.9 & 6.8 & 175.2 & 5.5 & 173.9 & 7.6 & -0.7 & -6.3 \\
\hline G16 & 819 & 0.39754 & 0.0186 & 0.0002 & 0.13265 & 0.0026 & 0.05298 & 0.001 & 327.9 & 12.2 & 118.8 & 4.5 & 126.5 & 7.1 & 6.1 & 3.9 \\
\hline G17 & 1195 & 0.07654 & 0.1498 & 0.0014 & 1.44295 & 0.0136 & 0.07013 & 0.00 & 932.2 & 10.5 & 899.9 & 17.6 & 906.9 & 15.9 & 0.8 & -0.9 \\
\hline G18 & 1771 & 0.5663 & 0.1882 & 0.0017 & 3.59155 & 0.0327 & 0.13651 & 0.0009 & 2183.4 & 13.4 & 1111.7 & 20.9 & 1547.7 & 19.8 & 28.2 & 23.2 \\
\hline G19 & 1071 & 0.13858 & 0.143 & 0.0013 & 1.3427 & 0.0137 & 0.06765 & 0.0005 & 857.8 & 10.5 & 861.7 & 17.0 & 864.4 & 15.7 & 0.3 & -2.2 \\
\hline $\mathrm{G} 25$ & 365 & 0.51182 & 0.2467 & 0.0023 & 2.81307 & 0.0373 & 0.08755 & 0.0007 & 1372.6 & 13.3 & 1421.6 & 25.8 & 1359.0 & 20.2 & -4.6 & -6.5 \\
\hline 626 & 76 & 1.37338 & 0.1657 & 0.0018 & 1.3184 & 0.0423 & 0.07373 & 0.0013 & 1034.1 & 24.8 & 988.1 & 22.5 & 853.8 & 28.5 & -15.7 & -40.4 \\
\hline G27 & 514 & 0.87692 & 0.0117 & 0.0002 & 0.09295 & 0.0028 & 0.05904 & & 568.6 & 27.0 & 74.7 & 4.0 & 90.2 & 7. & 17 & -9.8 \\
\hline $\mathrm{G} 28$ & 424 & 0.13048 & 0.1861 & 0.0017 & 1.80719 & 0.0251 & 0.07279 & 0.0006 & 1008.1 & 12.6 & 1100.4 & 21.0 & 1048.1 & 18.6 & -5.0 & -6.2 \\
\hline 629 & 622 & 0.1572 & 0.1157 & 0.0011 & 1.04963 & 0.0153 & 0.06782 & 0.0007 & 863.1 & 13.3 & 705.7 & 14.8 & 728.7 & 16.4 & 3.2 & -1.5 \\
\hline G30 & 667 & 0.50089 & 0.1272 & 0.0012 & 1.13322 & 0.016 & 0.06629 & 0.0006 & 15.5 & 12.3 & 771.9 & 15.8 & 769.3 & 16.4 & -0.3 & 2.7 \\
\hline G31 & 620 & 0.40178 & 0.015 & 0.0002 & 0.10563 & 0.0031 & 0.05192 & 0.0014 & 281.9 & 15.6 & 96.0 & 4.4 & 102.0 & 8.0 & 5.8 & 1.3 \\
\hline $\mathrm{G} 32$ & 1140 & 0.1279 & 0.1119 & 0.001 & 1.05669 & 0.0107 & 0.06936 & 0.0005 & 909.5 & 11.1 & 683.9 & & 732.2 & 14.6 & & 1.7 \\
\hline$G 33$ & 110 & 0.93669 & 0.572 & 0.0055 & 13.60916 & 0.3302 & 0.19597 & 0.0015 & 2792.9 & 16.5 & 2916.1 & 47.5 & 2722.9 & 25.5 & -7.1 & -8.4 \\
\hline $\mathrm{G} 34$ & 758 & & 0.1733 & 0.0016 & & 0.0227 & 0.07315 & & & 12 & & 19 & & 17.9 & 0.5 & -3.1 \\
\hline G35 & 323 & 0.43642 & 0.4739 & 0.0044 & 10.25258 & 0.1758 & 0.16158 & 0.0011 & 2472.3 & 15.1 & 2500.6 & 40.9 & 2457.8 & 23.8 & -1.7 & -3.0 \\
\hline $\mathrm{G} 36$ & 699 & 1.36459 & 0.1631 & 0.0015 & 1.58333 & 0.0176 & 0.07238 & 0.0005 & 996.6 & 11.6 & 973.8 & 18.8 & 963.7 & 17.1 & -1.0 & -36.6 \\
\hline G37 & 456 & & 0.1462 & & & & & & & & 879.7 & & 858.2 & & -2.5 & -5.3 \\
\hline$G 38$ & 152 & 0.6654 & 0.452 & 0.0043 & 7.46049 & 0.1367 & 0.13765 & 0.001 & 2197.8 & 15.6 & 2404.0 & 40.1 & 2168.3 & 24.1 & -10.9 & -9.3 \\
\hline 339 & 144 & 0.30129 & 0.196 & 0.0019 & & 0.0351 & & & & 16.4 & 1154.0 & & 1031.2 & & -11.9 & -9.9 \\
\hline
\end{tabular}


$\begin{array}{llllllllll}\text { G46 } & 675 & 0.36676 & 0.2773 & 0.0026 & 3.83734 & 0.0429 & 0.10042 & 0.0007\end{array}$

$\begin{array}{lllllllllll}\mathrm{G} 47 & 214 & 0.19511 & 0.4014 & 0.0038 & 6.44934 & 0.1062 & 0.12492 & 0.0009\end{array}$ $\begin{array}{lllllllll}559 & 0.81086 & 0.0853 & 0.0008 & 0.68177 & 0.0094 & 0.05978 & 0.0006\end{array}$ $\begin{array}{lllllllll}505 & 0.58738 & 0.4302 & 0.004 & 9.42611 & 0.1208 & 0.16244 & 0.0011\end{array}$ $\begin{array}{lllllllll}844 & 0.57739 & 0.1286 & 0.0012 & 1.17562 & 0.0138 & 0.06704 & 0.0006 \\ 155 & 0.48845 & 0.3077 & 0.003 & 3.85692 & 0.0816 & 0.09948 & 0.0009\end{array}$ $\begin{array}{lllllllll}155 & 0.48845 & 0.3077 & 0.003 & 3.85692 & 0.0816 & 0.09948 & 0.0009\end{array}$ $\begin{array}{lllllllll}1001 & 0.42152 & 0.2103 & 0.0019 & 2.45948 & 0.0241 & 0.08482 & 0.0006\end{array}$ $\begin{array}{llllllllll}817 & 0.38439 & 0.1494 & 0.0014 & 1.42838 & 0.0155 & 0.06981 & 0.0005\end{array}$ $\begin{array}{llllllllll}1109 & 0.19464 & 0.1415 & 0.0013 & 1.34921 & 0.0162 & 0.06901 & 0.0006\end{array}$ $\begin{array}{lllllllll}286 & 0.89501 & 0.1724 & 0.0017 & 1.65047 & 0.0282 & 0.0736 & 0.0007\end{array}$ $\begin{array}{lllllllll}410 & 0.72617 & 0.0879 & 0.0009 & 0.67896 & 0.0118 & 0.05844 & 0.0007\end{array}$ $\begin{array}{lllllllll}484 & 0.14553 & 0.1407 & 0.0013 & 1.31135 & 0.0198 & 0.0705 & 0.0007\end{array}$ $\begin{array}{llllllllll}1185 & 0.73859 & 0.0041 & 5 \mathrm{E}-05 & 0.02676 & 0.0009 & 0.04906 & 0.0016\end{array}$ $\begin{array}{lllllllll}490 & 0.6197 & 0.3751 & 0.0035 & 8.60245 & 0.1279 & 0.17159 & 0.0012\end{array}$ $\begin{array}{lllllllll}126 & 1.98962 & 0.0892 & 0.001 & 0.68206 & 0.0192 & 0.06477 & 0.0012\end{array}$ $\begin{array}{lllllllll}723 & 0.32379 & 0.1635 & 0.0015 & 1.55915 & 0.0198 & 0.07033 & 0.0006\end{array}$ $\begin{array}{llllllllll}387 & 0.73128 & 0.164 & 0.0016 & 1.56955 & 0.0244 & 0.07184 & 0.0007\end{array}$ $\begin{array}{lllllllll}1007 & 0.9762 & 0.0794 & 0.0008 & 0.62305 & 0.008 & 0.05759 & 0.0006\end{array}$ $\begin{array}{llllllllll}495 & 0.30935 & 0.1486 & 0.0014 & 1.34903 & 0.0203 & 0.06976 & 0.0007\end{array}$ $\begin{array}{lllllllll}372 & 0.31094 & 0.1395 & 0.0013 & 1.2457 & 0.0184 & 0.06704 & 0.0006\end{array}$ $\begin{array}{lllllllll}211 & 1.06962 & 0.1859 & 0.0018 & 1.75093 & 0.031 & 0.0738 & 0.0007\end{array}$ $\begin{array}{lllllllll}138 & 2.22088 & 0.0919 & 0.001 & 0.66502 & 0.0162 & 0.05973 & 0.001\end{array}$ $\begin{array}{lllllllll}183 & 0.23467 & 0.1126 & 0.0012 & 0.88156 & 0.0198 & 0.06265 & 0.0009\end{array}$ $\begin{array}{lllllllll}654 & 0.13117 & 0.0963 & 0.0009 & 0.81967 & 0.0107 & 0.06408 & 0.0006\end{array}$ $\begin{array}{lllllllll}294 & 0.86526 & 0.3204 & 0.003 & 4.42008 & 0.0675 & 0.10686 & 0.0009\end{array}$ $\begin{array}{lllllllll}1794 & 0.06435 & 0.1431 & 0.0013 & 1.36297 & 0.0128 & 0.06853 & 0.0005\end{array}$ $\begin{array}{lllllllll}584 & 0.51422 & 0.1742 & 0.0017 & 1.75659 & 0.0262 & 0.0772 & 0.0007\end{array}$ $\begin{array}{llllllllll}365 & 0.26472 & 0.5623 & 0.0052 & 20.97816 & 0.2879 & 0.28549 & 0.002\end{array}$ $\begin{array}{lllllllll}4276 & 0.20266 & 0.1068 & 0.001 & 1.09926 & 0.0093 & 0.07345 & 0.0005\end{array}$ $\begin{array}{lllllllll}2343 & 0.03447 & 0.3602 & 0.0033 & 6.32407 & 0.0669 & 0.12512 & 0.0009\end{array}$ $\begin{array}{lllllllll}195 & 0.49075 & 0.5982 & 0.0057 & 15.31426 & 0.2777 & 0.19775 & 0.0015\end{array}$ $\begin{array}{lllllllll}220 & 0.25465 & 0.186 & 0.0018 & 1.67264 & 0.0315 & 0.07487 & 0.0008\end{array}$ $\begin{array}{rrrrrrrrrr}511 & 0.58801 & 0.0181 & 0.0002 & 0.1336 & 0.0038 & 0.05686 & 0.0015\end{array}$ $\begin{array}{lllllllll}1504 & 0.33598 & 0.3082 & 0.0029 & 6.16964 & 0.0631 & 0.14646 & 0.0011\end{array}$ $\begin{array}{llllllllll}580 & 0.5793 & 0.1206 & 0.0011 & 1.12156 & 0.0148 & 0.0684 & 0.0006\end{array}$ $\begin{array}{llllllllll}746 & 0.44418 & 0.2418 & 0.0023 & 3.11217 & 0.0421 & 0.09655 & 0.0008\end{array}$ $\begin{array}{lllllllll}187 & 0.22943 & 0.1179 & 0.0012 & 0.96821 & 0.022 & 0.06393 & 0.0009\end{array}$ $\begin{array}{lllllllll}465 & 0.81966 & 0.1425 & 0.0014 & 1.29262 & 0.0208 & 0.06802 & 0.0007\end{array}$ $\begin{array}{llllllllll}924 & 0.27675 & 0.0807 & 0.0008 & 0.70938 & 0.0098 & 0.06522 & 0.0007\end{array}$ $\begin{array}{lllllllll}429 & 0.54538 & 0.0167 & 0.0002 & 0.44546 & 0.0084 & 0.20052 & 0.0032\end{array}$ $\begin{array}{llllllllll}648 & 0.28883 & 0.1458 & 0.0014 & 1.33596 & 0.0183 & 0.06854 & 0.0006\end{array}$ $\begin{array}{lllllllll}276 & 0.38655 & 0.4782 & 0.0046 & 10.54285 & 0.1993 & 0.16463 & 0.0013\end{array}$ $\begin{array}{lllllllllll}3161 & 0.6011 & 0.0673 & 0.0006 & 0.72614 & 0.0071 & 0.07772 & 0.0006\end{array}$ $\begin{array}{lllllllll}194 & 1.50988 & 0.1757 & 0.0017 & 1.64131 & 0.0317 & 0.07481 & 0.0008\end{array}$ $\begin{array}{lllllllll}1025 & 0.08862 & 0.3047 & 0.0028 & 4.93565 & 0.0544 & 0.11648 & 0.0009\end{array}$ $\begin{array}{lllllllll}860 & 0.43013 & 0.1365 & 0.0013 & 1.34683 & 0.0247 & 0.0721 & 0.0008\end{array}$ $\begin{array}{lllllllll}264 & 0.72845 & 0.1365 & 0.0013 & 1.34683 & 0.0247 & 0.0721 & 0.0008\end{array}$ $\begin{array}{lllllllll}264 & 0.72845 & 0.0923 & 0.001 & 0.68945 & 0.0151 & 0.05986 & 0.0009\end{array}$ $\begin{array}{llllllllll}438 & 0.49017 & 0.1931 & 0.0019 & 2.01556 & 0.0331 & 0.07822 & 0.0008 \\ 370 & 0.55449 & 0.0247 & 0.0003 & 0.16789 & 0.0045 & 0.05094 & 0.0012\end{array}$ $\begin{array}{lllllllll}370 & 0.55449 & 0.4839 & 0.0045 & 10.81895 & 0.1538 & 0.16536 & 0.0013\end{array}$ $\begin{array}{lllllrlll}321 & 0.31448 & 0.1759 & 0.0017 & 1.69086 & 0.0266 & 0.07448 & 0.0007\end{array}$ $\begin{array}{lllllllll}624 & 0.30549 & 0.3597 & 0.0034 & 6.1117 & 0.0935 & 0.12323 & 0.001\end{array}$ $\begin{array}{lllllllll}1163 & 0.20229 & 0.201 & 0.0019 & 3.0496 & 0.0333 & 0.11097 & 0.0009\end{array}$ $\begin{array}{llllllllll}266 & 0.6605 & 0.1414 & 0.0014 & 1.24448 & 0.021 & 0.0679 & 0.0007\end{array}$ $\begin{array}{lllllllll}437 & 1.78157 & 0.1535 & 0.0015 & 1.44533 & 0.0205 & 0.07023 & 0.0007\end{array}$ $\begin{array}{lll}1631.9 & 13.4 & 1577.6\end{array}$ $\begin{array}{rrr}595.6 & 11.2 & 2175.5 \\ 248.2 & 1.7 & 2306.8\end{array}$ $14.7 \quad 2306.8$ $11.5 \quad 779.6$ $\begin{array}{lll}1614.4 & 17.1 & 1729.2\end{array}$ $922.8-12.3 \quad 1230.1$ $899.0-11.8 \quad 897.4$ $\begin{array}{lll}1030.5 & 14.8 & 1025.2\end{array}$ 14.8025 .2 $943.0-13.8 \quad 848.3$ $\begin{array}{llr}150.7 & 11.3 & 26.1\end{array}$ $766.9-15.6 \quad 2053.4$
-150.5

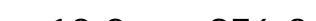
\begin{tabular}{lll}
981.4 & 13.8 & 976.3 \\
\hline & 13.8
\end{tabular} 
$\begin{array}{lllllllll}602 & 1.39601 & 0.3513 & 0.0033 & 5.75662 & 0.0722 & 0.12006 & 0.001 & 1957.1\end{array}$ $\begin{array}{lllllllll}993 & 0.30006 & 0.1612 & 0.0015 & 1.5981 & 0.0188 & 0.0718 & 0.0006\end{array}$ 1957.1
980.3 $\begin{array}{lllllllll}397 & 1.05373 & 0.0183 & 0.0002 & 0.1213 & 0.004 & 0.05076 & 0.0016\end{array}$ $\begin{array}{lllllllll}463 & 0.7839 & 0.2217 & 0.0021 & 2.4926 & 0.0357 & 0.08425 & 0.0008\end{array}$ $\begin{array}{lllllllll}381 & 0.52352 & 0.0149 & 0.0002 & 0.0951 & 0.0035 & 0.04823 & 0.0017\end{array}$ $\begin{array}{llllllllll}180 & 1.62061 & 0.0899 & 0.0009 & 0.67479 & 0.0162 & 0.05901 & 0.001\end{array}$ $\begin{array}{lllllllll}560 & 0.13489 & 0.3286 & 0.0031 & 5.16184 & 0.0776 & 0.11366 & 0.001\end{array}$ $\begin{array}{lllllllll}450 & 0.50705 & 0.138 & 0.0014 & 1.21758 & 0.0219 & 0.0673 & 0.0008\end{array}$ $\begin{array}{lllllllll}353 & 0.32618 & 0.3491 & 0.0033 & 5.19077 & 0.0785 & 0.113 & 0.001\end{array}$ $\begin{array}{llllllllll}325 & 1.1078 & 0.0424 & 0.0005 & 0.46987 & 0.0102 & 0.08386 & 0.0014\end{array}$ $\begin{array}{llll}185 & 0.49377 & 0.3785 \quad 0.0038 & 0.07\end{array}$ $\begin{array}{llllllll}1804 & 0.26101 & 0.4575 & 0.0043 & 10.61227 & 0.1142 & 0.16321 & 0.0013\end{array}$ $\begin{array}{lllllllll}690 & 0.63915 & 0.2917 & 0.0027 & 3.94308 & 0.0511 & 0.09925 & 0.0008\end{array}$

$\begin{array}{rr}16.0 & 1940.7\end{array}$

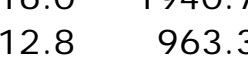

$33.4 \quad 1939.9$

$\begin{array}{lll}23.4 & 0.0 & -30.8\end{array}$

SAMPLE: MAK4

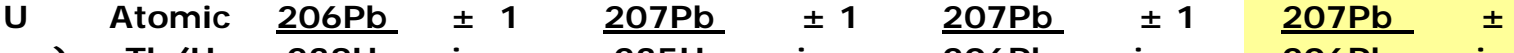

$\underline{206 \mathrm{~Pb}} \quad \pm 2 \quad \underline{207 \mathrm{~Pb}}$

2380

sigma

$\begin{array}{llllllllll}823 & 0.1 & 0.1315 & 0.0014 & 1.28269 & 0.0171 & 0.07074 & 0.0005\end{array}$

$\begin{array}{lllllllll}2172 & 0.0 & 0.0886 & 0.0009 & 0.66881 & 0.0057 & 0.05448 & 0.0003\end{array}$

$\begin{array}{lllllllll}703 & 0.3 & 0.0331 & 0.0004 & 0.23507 & 0.0037 & 0.05023 & 0.0006\end{array}$

$\begin{array}{lllllllll}1381 & 0.1 & 0.1173 & 0.0012 & 0.97943 & 0.0101 & 0.05998 & 0.0004\end{array}$ $\begin{array}{lllllll}0.08 & 0.0009 & 0.61696 & 0.0072 & 0.05585 & 0.0004\end{array}$ $\begin{array}{lllllll}0.0243 & 0.0003 & 0.15812 & 0.0041 & 0.06957 & 0.0015\end{array}$

$\begin{array}{lllllllll}194 & 0.7 & 0.0243 & 0.0003 & 0.15812 & 0.0041 & 0.0595 & 0.0015 \\ 633 & 0.4 & 0.4466 & 0.0047 & 9.45768 & 0.121 & 0.15253 & 0.0009\end{array}$

$\begin{array}{lllllllll}1280 & 0.2 & 0.1385 & 0.0015 & 1.3087 & 0.0139 & 0.068 & 0.0004\end{array}$ $\begin{array}{rrrrrrrr}355 & 0.4 & 0.1821 & 0.002 & 1.5575 & 0.0241 & 0.07741 & 0.0006 \\ 541 & 0.4 & 0.0157 & 0.0002 & 0.10552 & 0.0034 & 0.04975 & 0.0014\end{array}$ $\begin{array}{llllll}0.3166 & 0.0035 & 4.75192 & 0.0694 & 0.10878 & 0.0008\end{array}$ $\begin{array}{lll}957.8 & 20.0 & 785.5 \\ 1779.1 & 13.3 & 1773.3\end{array}$ $\begin{array}{llr}1779.1 & 13.3 & 1773.3\end{array}$ $\begin{array}{lll}747.9 & 19.8 & 796.6\end{array}$ $\begin{array}{rrrrrrrr}840 & 0.1 & 0.1315 & 0.0015 & 1.14405 & 0.02 & 0.06419 & 0.0006 \\ 694 & 0.1 & 0.0969 & 0.0011 & 0.81779 & 0.0139 & 0.06204 & 0.0006\end{array}$ $\begin{array}{llllrlll}3229 & 0.4 & 0.0073 & 9 \mathrm{E}-05 & 0.0526 & 0.0008 & 0.05149 & 0.0006\end{array}$ $\begin{array}{lllllllll}829 & 0.1 & 0.1531 & 0.0018 & 1.4345 & 0.0211 & 0.06754 & 0.0006\end{array}$ $\begin{array}{lllllllll}209 & 0.6 & 0.0319 & 0.0005 & 0.20864 & 0.006 & 0.07041 & 0.0016\end{array}$ $\begin{array}{lllllllll}423 & 0.4 & 0.4795 & 0.0056 & 8.76077 & 0.1505 & 0.15159 & 0.0013\end{array}$ $\begin{array}{lllllllll}798 & 0.2 & 0.0912 & 0.0011 & 0.7786 & 0.0162 & 0.06112 & 0.0007\end{array}$ $\begin{array}{lllllllll}2587 & 0.9 & 0.0034 & 5 \mathrm{E}-05 & 0.02305 & 0.0008 & 0.0485 & 0.0016\end{array}$ $\begin{array}{lllllllll}212 & 0.7 & 0.0296 & 0.0005 & 0.19745 & 0.0064 & 0.06817 & 0.0017\end{array}$ $\begin{array}{lllllllll}524 & 0.2 & 0.1357 & 0.0016 & 1.21918 & 0.0241 & 0.06722 & 0.0007\end{array}$ $\begin{array}{lllllllll}1237 & 0.1 & 0.1575 & 0.0019 & 1.51468 & 0.0274 & 0.06915 & 0.0007\end{array}$ $\begin{array}{lllllllll}610 & 0.2 & 0.1296 & 0.0016 & 1.10646 & 0.0237 & 0.06252 & 0.0008\end{array}$ $\begin{array}{rrrrrrrrr}977 & 0.6 & 0.1432 & 0.0017 & 1.37579 & 0.0268 & 0.069 & 0.0008\end{array}$ $\begin{array}{rrrrrrrr}511 & 0.3 & 0.2186 & 0.0027 & 2.5943 & 0.0584 & 0.08498 & 0.001 \\ 2351 & 0.7 & 0.106 & 0.0013 & 0.88695 & 0.0177 & 0.05979 & 0.0007\end{array}$ $\begin{array}{lllllllll}834 & 0.3 & 0.1161 & 0.0015 & 1.06534 & 0.024 & 0.06769 & 0.0009\end{array}$ 
$\begin{array}{lllllllll}\text { G33 } & 169 & 0.5 & 0.0211 & 0.0004 & 0.14108 & 0.005 & 0.07148 & 0.002\end{array}$ $\begin{array}{lllllllll}497 & 0.1 & 0.4651 & 0.0059 & 6.73891 & 0.1672 & 0.13308 & 0.0018\end{array}$ $\begin{array}{lllllllll}1071 & 0.1 & 0.0869 & 0.0011 & 0.68361 & 0.0169 & 0.05821 & 0.0009\end{array}$ $\begin{array}{lllllllll}662 & 0.4 & 0.0189 & 0.0003 & 0.12636 & 0.0039 & 0.05252 & 0.0011\end{array}$ $\begin{array}{lllllllll}1357 & 0.1 & 0.14 & 0.0018 & 1.26603 & 0.0339 & 0.06549 & 0.001\end{array}$ $\begin{array}{lllllllll}1218 & 0.4 & 0.143 & 0.0019 & 1.3086 & 0.0361 & 0.0655 & 0.0011\end{array}$ $\begin{array}{llllllll}554 & 0.3 & 0.287 & 0.0038 & 4.2252 & 0.1216 & 0.12558 & 0.002\end{array}$ $\begin{array}{lllllllll}761 & 0.2 & 0.3499 & 0.0046 & 5.34437 & 0.1604 & 0.10952 & 0.0018\end{array}$ $\begin{array}{lllllllll}866 & 0.4 & 0.0254 & 0.0004 & 0.19269 & 0.0059 & 0.05532 & 0.0011\end{array}$ $\begin{array}{lllllllll}782 & 0.2 & 0.1046 & 0.0014 & 0.86912 & 0.0274 & 0.05947 & 0.0011\end{array}$ $\begin{array}{lllllllll}1095 & 0.9 & 0.0166 & 0.0002 & 0.11259 & 0.0038 & 0.04875 & 0.0011\end{array}$ $\begin{array}{lllllllll}303 & 0.6 & 0.0126 & 0.0002 & 0.08348 & 0.0033 & 0.07008 & 0.0022\end{array}$ $\begin{array}{lllllllll}2246 & 1.0 & 0.0164 & 0.0002 & 0.11747 & 0.0037 & 0.05181 & 0.0011\end{array}$ $\begin{array}{llllllll}4587 & 1.2 & 0.0149 & 0.0002 & 0.11425 & 0.0036 & 0.05536 & 0.0011\end{array}$

$\begin{array}{rrr}971.2 & 57.9 & 134.6 \\ 2139.0 & 23.8 & 2462.2 \\ 537.7 & 32.0 & 537.4 \\ 308.1 & 49.4 & 120.4 \\ 790.1 & 32.7 & 844.6 \\ 790.4 & 33.7 & 861.6 \\ 2037.0 & 28.3 & 1626.5 \\ 1791.4 & 29.6 & 1934.1 \\ 425.2 & 44.0 & 161.4 \\ 584.3 & 39.4 & 641.5 \\ 135.8 & 54.5 & 106.3 \\ 930.7 & 64.4 & 80.8 \\ 277.0 & 46.4 & 105.0 \\ 426.8 & 43.5 & 95.3\end{array}$

$\begin{array}{rrrc}4.6 & 134.0 & 10.1 & -0.4 \\ 75.6 & 2077.7 & 293.0 & -18.5 \\ 14.3 & 529.0 & 33.8 & -1.6 \\ 3.6 & 120.8 & 7.9 & 0.3 \\ 23.4 & 830.6 & 66.6 & -1.7 \\ 24.1 & 849.5 & 70.8 & -1.4 \\ 48.4 & 1678.9 & 221.0 & 3.1 \\ 59.2 & 1876.0 & 282.5 & -3.1 \\ 4.6 & 178.9 & 11.9 & 9.8 \\ 18.2 & 635.1 & 54.3 & -1.0 \\ 3.3 & 108.3 & 7.7 & 1.9 \\ 3.0 & 81.4 & 6.8 & 0.7 \\ 3.2 & 112.8 & 7.6 & 6.9 \\ 2.8 & 109.8 & 7.3 & 13.2\end{array}$

SAMPLE: MAK 9

Apparent Ages (Ma)

U Atomic $\frac{206 \mathrm{~Pb}}{ \pm 1} \quad \underline{207 \mathrm{~Pb}} \quad \pm 1 \quad \underline{207 \mathrm{~Pb}} \quad \pm 1 \quad \underline{207 \mathrm{~Pb}}$

$\frac{206 \mathrm{~Pb}}{238 \mathrm{~b}} \pm 2 \quad \underline{207 \mathrm{~Pb}}$

$\underline{206 / 238} \quad \underline{207 / 206}$

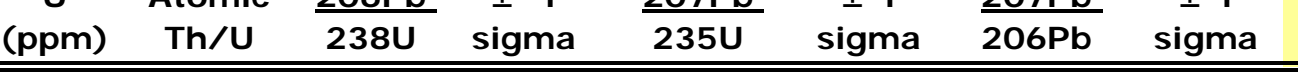

$206 \mathrm{~Pb}$ sigma $\frac{206 \mathrm{~Pb}}{238 \mathrm{U}}$

gma $235 \mathrm{U}$ sigm

$\begin{array}{lllllllll}317 & 0.4 & 0.1369 & 0.0012 & 1.22442 & 0.0172 & 0.06792 & 0.0009\end{array}$

$4.6 \quad 202.9$

$866.1 \quad 14.9 \quad 827.1$

$\begin{array}{rrrrr}3.6 & 201.8 & 5.9 & -0.5 & -20.7 \\ 13.7 & 811.8 & 17.7 & -1.9 & -3.3\end{array}$

$\begin{array}{lllllllll}317 & 0.2 & 0.3296 & 0.0029 & 4.59284 & 0.0867 & 0.10654 & 0.0013\end{array}$

$\begin{array}{llr}1741.0 & 21.0 & 1836.5\end{array}$

$\begin{array}{lllll}13.7 & 811.8 & 17.7 & -1.9 & -3.3 \\ 28.4 & 1748.0 & 25.9 & -5.1 & -5.4\end{array}$

$\begin{array}{lllllll}0.0 & 0.1854 & 0.0016 & 2.34451 & 0.0253 & 0.09061 & 0.001\end{array}$

$1438.4 \quad 21.0 \quad 1836.5$

$\begin{array}{lllll}28.4 & 1748.0 & 25.9 & -5.1 & -5.4 \\ 17.5 & 1225.9 & 20.0 & 10.6 & 7.3\end{array}$

\begin{tabular}{rrrrrrrrrrrrrrrr}
294 & 0.6 & 0.0165 & 0.0002 & 0.10736 & 0.003 & 0.04952 & 0.0014 & 172.5 & 9.1 & 105.2 & 2.2 & 103.5 & 6.3 & -1.6 & -52.3 \\
\hline
\end{tabular}

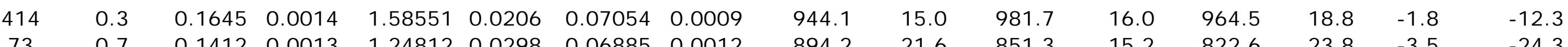

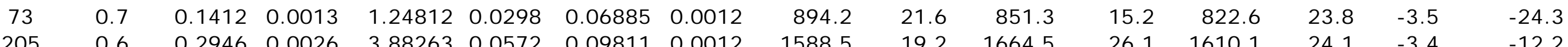

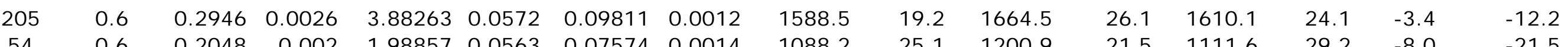

$\begin{array}{rrrrrrrrrrrrrrrr}54 & 0.6 & 0.2048 & 0.002 & 1.98857 & 0.0563 & 0.07574 & 0.0014 & 1088.2 & 25.1 & 1200.9 & 21.5 & 1111.6 & 29.2 & -8.0 & -21.5 \\ 466 & 0.1 & 0.3019 & 0.0027 & 4.92084 & 0.0585 & 0.11771 & 0.0013 & 1921.7 & 19.5 & 1700.8 & 26.5 & 1805.8 & 24.2 & 5.8 & 4.8\end{array}$

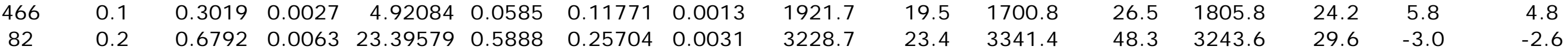

$\begin{array}{llllllllllllllll}289 & 0.9 & 0.2681 & 0.0024 & 3.30159 & 0.0519 & 0.09284 & 0.0012 & 1484.6 & 19.5 & 1531.2 & 24.8 & 1481.4 & 24.3 & -3.4 & -26.1\end{array}$

$\begin{array}{llllllllllllllll}291 & 0.2 & 0.1431 & 0.0013 & 1.38527 & 0.02 & 0.07289 & 0.0009 & 1010.9 & 16.7 & 861.9 & 14.7 & 882.7 & 19.0 & 2.3 & -9.9 \\ 8 & 0.1 & 0.4723 & 0.0042 & 8.97333 & 0.148 & 0.1400 & 0.0016 & 2228 & 20.8 & 2493.8 & 37.2 & 2335.3 & 26.8 & -6.8 & -6.8\end{array}$

$\begin{array}{rrrrrrrrrrrrrrrr}878 & 0.1 & 0.4723 & 0.0042 & 8.97333 & 0.1087 & 0.14009 & 0.0016 & 2228.3 & 20.8 & 2493.8 & 37.2 & 2335.3 & 26.8 & -6.8 & -6.8 \\ 247 & 0.3 & 0.1343 & 0.0013 & 1.45027 & 0.0269 & 0.08305 & 0.0012 & 1270.4 & 21.7 & 812.5 & 14.5 & 910.0 & 21.8 & 10.7 & 5.2\end{array}$

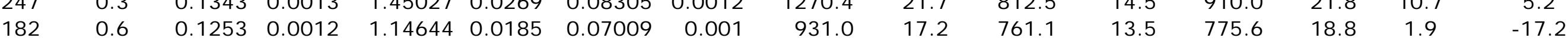

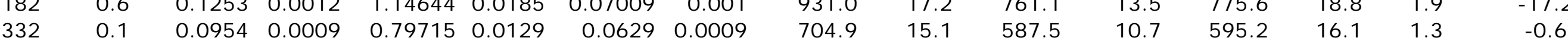

$\begin{array}{rrrlllllllllllll}366 & 0.9 & 0.1607 & 0.0015 & 1.59411 & 0.0262 & 0.07512 & 0.001 & 1071.7 & 18.5 & 960.6 & 16.9 & 967.9 & 21.4 & 0.8 & -35.2\end{array}$

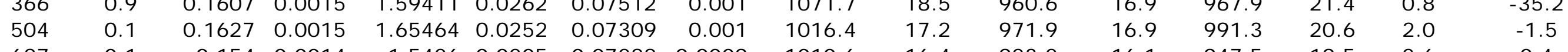

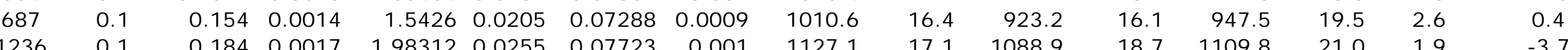

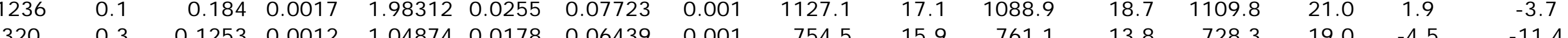

$\begin{array}{rrrrrrrrrrrrrrrr}320 & 0.3 & 0.1253 & 0.0012 & 1.04874 & 0.0178 & 0.06439 & 0.001 & 754.5 & 15.9 & 761.1 & 13.8 & 728.3 & 19.0 & -4.5 & -11.4 \\ 233 & 0.9 & 0.1618 & 0.0016 & 1.48642 & 0.0256 & 0.07023 & 0.001 & 935.1 & 17.9 & 966.5 & 17.4 & 924.9 & 21.8 & -4.5 & -54.6\end{array}$

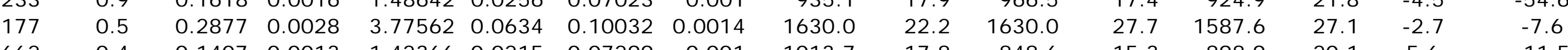

$\begin{array}{rrrrrrrrrrrrrrrr}662 & 0.4 & 0.1407 & 0.0013 & 1.42366 & 0.0215 & 0.07299 & 0.001 & 1013.7 & 17.8 & 848.6 & 15.3 & 898.9 & 20.1 & 5.6 & -11.5 \\ 372 & 0.5 & 0.4546 & 0.0043 & 9.88655 & 0.1613 & 0.16092 & 0.0021 & 2465.3 & 24.7 & 2415.7 & 38.7 & 2424.3 & 30.3 & 0.4 & -0.6\end{array}$

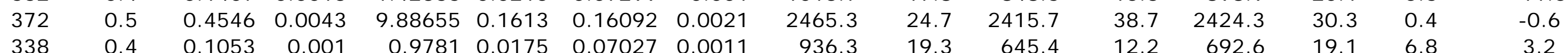

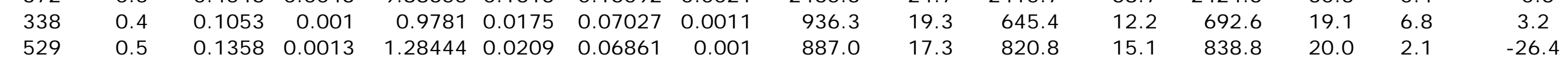

$\begin{array}{llllllllllllllll}88 & 0.5 & 0.0183 & 0.0002 & 0.12889 & 0.0061 & 0.05399 & 0.0025 & 370.6 & 28.6 & 116.8 & 3.1 & 123.1 & 11.7 & 5.1 & -21.5\end{array}$

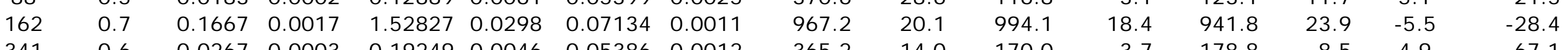

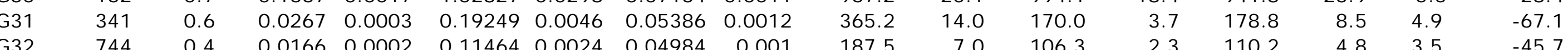




\begin{tabular}{|c|c|c|c|c|c|c|c|c|c|c|c|c|c|c|c|c|}
\hline G33 & 1237 & 0.1 & 0.4364 & 0.0043 & 8.91831 & 0.1476 & 0.1479 & 0.0021 & 2321.8 & 26.6 & 2334.5 & 38.7 & 2329.7 & 32.1 & -0.2 & -0.7 \\
\hline G34 & 196 & 0.4 & 0.1714 & 0.0017 & 1.86715 & 0.0374 & 0.08458 & 0.0014 & 1305.9 & 24.2 & 1019.5 & 19.2 & 1069.5 & 26.2 & 4.7 & -3.6 \\
\hline G35 & 1180 & 0.0 & 0.0778 & 0.0008 & 0.61947 & 0.0111 & 0.05719 & 0.0009 & 498.9 & 12.9 & 483.2 & 9.5 & 489.5 & 14.8 & 1.3 & -1.5 \\
\hline G36 & 245 & 0.5 & 0.1071 & 0.0012 & 0.9923 & 0.0287 & 0.07216 & 0.0016 & 990.5 & 28.9 & 655.6 & 13.8 & 699.9 & 26.6 & 6.3 & -5.6 \\
\hline G37 & 103 & 1.2 & 0.0867 & 0.0009 & 0.67062 & 0.0195 & 0.05835 & 0.0014 & 542.9 & 20.7 & 535.9 & 11.3 & 521.1 & 22.4 & -2.8 & -114.5 \\
\hline G38 & 173 & 0.5 & 0.0356 & 0.0005 & 0.2471 & 0.0119 & 0.05351 & 0.0024 & 350.5 & 26.3 & 225.6 & 6.0 & 224.2 & 19.6 & -0.6 & -11.6 \\
\hline G39 & 694 & 0.4 & 0.0244 & 0.0003 & 0.16857 & 0.004 & 0.05176 & 0.0011 & 274.8 & 10.8 & 155.4 & 3.5 & 158.2 & 7.6 & 1.8 & -29.3 \\
\hline G40 & 340 & 0.4 & 0.1799 & 0.0019 & 1.73123 & 0.0358 & 0.07337 & 0.0013 & 1024.2 & 22.3 & 1066.5 & 20.4 & 1020.2 & 26.4 & -4.5 & -13.4 \\
\hline G41 & 370 & 0.5 & 0.5314 & 0.0055 & 12.06479 & 0.2453 & 0.17278 & 0.0028 & 2584.8 & 31.0 & 2747.5 & 46.1 & 2609.5 & 36.7 & -5.3 & -5.3 \\
\hline G42 & 819 & 0.3 & 0.1409 & 0.0015 & 1.48263 & 0.0291 & 0.07608 & 0.0013 & 1097.2 & 22.9 & 849.9 & 16.6 & 923.3 & 24.2 & 7.9 & 8.2 \\
\hline G43 & 550 & 0.1 & 0.1487 & 0.0016 & 1.37004 & 0.0294 & 0.0694 & 0.0013 & 910.7 & 21.8 & 893.6 & 17.6 & 876.2 & 25.0 & -2.0 & -4.2 \\
\hline G44 & 319 & 0.2 & 0.2255 & 0.0024 & 2.53786 & 0.0633 & 0.08929 & 0.0017 & 1410.4 & 29.2 & 1310.7 & 25.5 & 1283.0 & 32.8 & -2.2 & -1.9 \\
\hline G45 & 134 & 0.2 & 0.5307 & 0.0066 & 11.75941 & 0.6094 & 0.17658 & 0.0039 & 2621.0 & 41.9 & 2744.4 & 55.4 & 2585.4 & 48.1 & -6.1 & -3.3 \\
\hline G46 & 884 & 0.0 & 0.4074 & 0.0043 & 9.34937 & 0.1964 & 0.16473 & 0.0029 & 2504.8 & 33.0 & 2203.0 & 39.2 & 2372.9 & 37.8 & 7.2 & 6.7 \\
\hline G47 & 693 & 0.3 & 0.2043 & 0.0022 & 2.31713 & 0.0515 & 0.08439 & 0.0016 & 1301.6 & 27.3 & 1198.3 & 23.3 & 1217.5 & 30.6 & 1.6 & 0.3 \\
\hline G48 & 144 & 0.5 & 0.029 & 0.0004 & 0.36269 & 0.0128 & 0.09409 & 0.003 & 1509.9 & 49.5 & 184.1 & 4.8 & 314.2 & 19.0 & 41.4 & 42.5 \\
\hline G49 & 364 & 0.3 & 0.1741 & 0.0019 & 1.66157 & 0.0406 & 0.07403 & 0.0015 & 1042.3 & 26.1 & 1034.8 & 20.8 & 994.0 & 29.6 & -4.1 & -7.2 \\
\hline G50 & 157 & 0.4 & 0.1197 & 0.0015 & 0.97989 & 0.0409 & 0.06359 & 0.002 & 728.0 & 33.2 & 729.0 & 17.3 & 693.5 & 35.7 & -5.1 & -38.7 \\
\hline G51 & 107 & 0.7 & 0.1096 & 0.0013 & 0.92174 & 0.0301 & 0.06551 & 0.0017 & 790.8 & 28.7 & 670.5 & 14.9 & 663.3 & 28.9 & -1.1 & -31.2 \\
\hline G52 & 411 & 0.4 & 0.163 & 0.0018 & 1.49002 & 0.0371 & 0.07197 & 0.0015 & 985.1 & 25.9 & 973.3 & 19.8 & 926.3 & 29.2 & -5.1 & -3.8 \\
\hline G53 & 350 & 0.1 & 0.1195 & 0.0013 & 1.00789 & 0.0264 & 0.06509 & 0.0014 & 777.2 & 23.6 & 727.8 & 15.4 & 707.8 & 25.6 & -2.8 & -5.8 \\
\hline G54 & 86 & 0.4 & 0.0165 & 0.0003 & 0.10865 & 0.011 & 0.05086 & 0.005 & 234.5 & 41.5 & 105.5 & 4.4 & 104.7 & 21.4 & -0.7 & -9.9 \\
\hline G55 & 332 & 0.3 & 0.0929 & 0.001 & 0.70126 & 0.0192 & 0.05923 & 0.0013 & 575.6 & 20.1 & 572.4 & 12.5 & 539.5 & 22.3 & -6.1 & -9.7 \\
\hline G56 & 535 & 0.1 & 0.1455 & 0.0016 & 1.32185 & 0.034 & 0.06782 & 0.0014 & 863.1 & 24.9 & 875.4 & 18.2 & 855.3 & 28.2 & -2.3 & -2.7 \\
\hline G57 & 305 & 0.6 & 0.1899 & 0.0021 & 1.89714 & 0.0512 & 0.07683 & 0.0017 & 1116.8 & 29.5 & 1120.6 & 23.1 & 1080.1 & 33.1 & -3.7 & -25.2 \\
\hline G58 & 484 & 0.2 & 0.0925 & 0.001 & 0.74391 & 0.0203 & 0.06066 & 0.0014 & 627.2 & 21.3 & 570.3 & 12.5 & 564.7 & 22.5 & -1.0 & -4.2 \\
\hline G59 & 585 & 0.8 & 0.0196 & 0.0003 & 0.13247 & 0.0057 & 0.05325 & 0.0021 & 339.5 & 22.8 & 124.9 & 3.5 & 126.3 & 10.8 & 1.1 & -68.4 \\
\hline G60 & 767 & 1.3 & 0.3918 & 0.0044 & 8.78162 & 0.2337 & 0.16232 & 0.0035 & 2480.0 & 40.2 & 2131.0 & 40.6 & 2315.6 & 44.4 & 8.0 & -38.8 \\
\hline G61 & 346 & 0.9 & 0.1352 & 0.0015 & 1.17664 & 0.0337 & 0.06769 & 0.0016 & 859.1 & 27.1 & 817.3 & 17.6 & 789.7 & 29.4 & -3.5 & -32.1 \\
\hline G62 & 914 & 0.3 & 0.252 & 0.0028 & 3.80498 & 0.1043 & 0.11047 & 0.0025 & 1807.2 & 37.8 & 1448.7 & 29.3 & 1593.8 & 40.4 & 9.1 & 7.4 \\
\hline G63 & 673 & 0.1 & 0.1192 & 0.0014 & 1.05449 & 0.0298 & 0.06464 & 0.0015 & 762.6 & 25.1 & 725.8 & 15.7 & 731.1 & 27.2 & 0.7 & -1.1 \\
\hline G64 & 106 & 1.0 & 0.015 & 0.0003 & 0.10067 & 0.0095 & 0.05386 & 0.0049 & 365.2 & 56.1 & 95.7 & 4.0 & 97.4 & 19.1 & 1.7 & -128.9 \\
\hline G65 & 390 & 1.0 & 0.3464 & 0.004 & 5.16835 & 0.1518 & 0.11385 & 0.0027 & 1861.8 & 39.9 & 1917.5 & 38.1 & 1847.4 & 44.7 & -3.8 & -17.6 \\
\hline G66 & 263 & 0.3 & 0.478 & 0.0055 & 9.08386 & 0.2769 & 0.14673 & 0.0035 & 2308.2 & 43.1 & 2518.5 & 48.1 & 2346.5 & 48.5 & -7.3 & -6.4 \\
\hline G67 & 600 & 0.0 & 0.0921 & 0.0011 & 0.7156 & 0.023 & 0.0587 & 0.0015 & 556.0 & 22.5 & 567.9 & 13.1 & 548.1 & 25.0 & -3.6 & -1.9 \\
\hline G68 & 278 & 0.2 & 0.0938 & 0.0011 & 0.73096 & 0.0253 & 0.05934 & 0.0017 & 579.6 & 24.9 & 578.2 & 13.5 & 557.1 & 27.1 & -3.8 & -13.5 \\
\hline G69 & 1093 & 0.1 & 0.1672 & 0.002 & 1.65591 & 0.0525 & 0.07249 & 0.0019 & 999.7 & 32.8 & 996.9 & 22.0 & 991.8 & 36.1 & -0.5 & 1.7 \\
\hline G70 & 660 & 0.1 & 0.029 & 0.0004 & 0.20926 & 0.0074 & 0.05345 & 0.0016 & 347.9 & 17.7 & 184.0 & 4.6 & 192.9 & 11.7 & 4.6 & -1.7 \\
\hline G71 & 267 & 0.4 & 0.5502 & 0.0066 & 13.4947 & 0.4606 & 0.18999 & 0.005 & 2742.1 & 50.1 & 2825.9 & 54.9 & 2714.9 & 55.2 & -4.1 & -2.5 \\
\hline G72 & 366 & 0.5 & 0.148 & 0.0018 & 1.28775 & 0.0448 & 0.06619 & 0.0018 & 812.4 & 31.3 & 889.9 & 20.3 & 840.3 & 35.6 & -5.9 & -19.5 \\
\hline G73 & 570 & 0.8 & 0.0121 & 0.0002 & 0.0786 & 0.0037 & 0.04905 & 0.0021 & 150.2 & 12.3 & 77.3 & 2.4 & 76.8 & 7.2 & -0.6 & -77.5 \\
\hline G74 & 139 & 0.4 & 0.0158 & 0.0003 & 0.10347 & 0.0093 & 0.04905 & 0.0042 & 150.2 & 24.3 & 101.2 & 4.0 & 100.0 & 17.6 & -1.2 & -17.2 \\
\hline G75 & 210 & 0.8 & 0.331 & 0.0041 & 4.45715 & 0.1624 & 0.10494 & 0.003 & 1713.3 & 46.9 & 1843.3 & 39.4 & 1723.0 & 51.8 & -7.0 & -15.1 \\
\hline G76 & 327 & 0.3 & 0.1666 & 0.0021 & 2.04978 & 0.0819 & 0.0955 & 0.0029 & 1538.0 & 48.3 & 993.1 & 23.6 & 1132.2 & 46.1 & 12.3 & 10.4 \\
\hline G77 & 173 & 0.4 & 0.0152 & 0.0002 & 0.11745 & 0.0066 & 0.05967 & 0.0031 & 591.6 & 46.5 & 97.3 & 3.1 & 112.8 & 12.3 & 13.7 & -7.0 \\
\hline G78 & 311 & 0.1 & 0.0475 & 0.0006 & 0.32174 & 0.013 & 0.05222 & 0.0018 & 295.1 & 17.5 & 299.1 & 7.7 & 283.2 & 18.9 & -5.6 & -17.8 \\
\hline G79 & 267 & 0.7 & 0.0926 & 0.0012 & 0.68557 & 0.0269 & 0.05698 & 0.0018 & 490.8 & 24.8 & 570.7 & 14.0 & 530.1 & 29.3 & -7.7 & -31.1 \\
\hline G80 & 90 & 1.5 & 0.3012 & 0.0038 & 3.81011 & 0.1582 & 0.09622 & 0.003 & 1552.1 & 49.3 & 1697.1 & 38.2 & 1594.9 & 54.4 & -6.4 & -46.8 \\
\hline G81 & 21 & 1.0 & 0.1441 & 0.0022 & 1.1602 & 0.0766 & 0.0625 & 0.0031 & 691.3 & 50.2 & 867.6 & 25.3 & 782.0 & 59.0 & -10.9 & -40.2 \\
\hline G82 & 103 & 1.7 & 0.0924 & 0.0013 & 0.6627 & 0.0311 & 0.05436 & 0.0021 & 386.0 & 24.4 & 569.4 & 14.9 & 516.3 & 33.5 & -10.3 & -1006.9 \\
\hline G83 & 462 & 0.1 & 0.1449 & 0.0018 & 1.32188 & 0.0522 & 0.07045 & 0.0022 & 941.5 & 38.9 & 872.2 & 20.9 & 855.3 & 40.5 & -2.0 & 0.3 \\
\hline G84 & 183 & 0.9 & 0.342 & 0.0044 & 4.78601 & 0.1977 & 0.10701 & 0.0034 & 1749.1 & 53.0 & 1896.1 & 42.4 & 1782.4 & 58.1 & -6.4 & -15.1 \\
\hline G85 & 709 & 0.1 & 0.2939 & 0.0038 & 4.52107 & 0.1804 & 0.11267 & 0.0036 & 1842.9 & 54.4 & 1660.8 & 37.7 & 1734.9 & 57.4 & 4.3 & 3.9 \\
\hline G86 & 158 & 0.6 & 0.157 & 0.0021 & 1.32222 & 0.0575 & 0.06571 & 0.0023 & 797.2 & 38.0 & 940.2 & 23.3 & 855.5 & 43.9 & -9.9 & -17.8 \\
\hline G87 & 211 & 0.3 & 0.4173 & 0.0054 & 8.0253 & 0.3371 & 0.14966 & 0.0049 & 2342.0 & 60.2 & 2248.3 & 49.5 & 2233.9 & 64.4 & -0.6 & 0.1 \\
\hline
\end{tabular}




\begin{tabular}{|c|c|c|c|c|c|c|c|c|c|c|c|c|c|c|c|c|}
\hline & & & & & & & & & & & & & & & & \\
\hline$\overline{\mathrm{G} 1}$ & 749 & $\overline{c 0.5}$ & 0.6195 & $\overline{c 0.0049}$ & 21.1634 & $\bar{~} 0.7792$ & $\bar{~} 0.2554$ & 0.0017 & 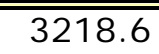 & 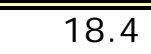 & 3108.1 & $\bar{~} \overline{44.2}$ & 3146.1 & 225.5 & 1.2 & 3.4 \\
\hline $\mathrm{G} 2$ & 8 & 1.8 & 0.1930 & 0.0022 & 2.4957 & 0.1429 & 0.0952 & 0.0018 & 1532.8 & 34.7 & 1137.3 & 29.6 & 1270.8 & 37.2 & 10.5 & 25.8 \\
\hline G3 & 51 & 0.3 & 0.1463 & 0.0011 & 1.3363 & 0.0283 & 0.0682 & 0.0006 & 874.6 & 16.3 & 880.4 & 17.9 & 861.6 & 19.5 & -2.2 & -0.7 \\
\hline G4 & 213 & 0.5 & 0.3358 & 0.0023 & 5.7236 & 0.0774 & 0.1265 & 0.0007 & 2049.3 & 15.1 & 1866.5 & 27.6 & 1934.9 & 20.6 & 3.5 & 8.9 \\
\hline G5 & 335 & 0.0 & 0.3103 & 0.0021 & 5.2486 & 0.0552 & 0.1195 & 0.0006 & 1948.0 & 14.5 & 1742.2 & 26.2 & 1860.5 & 20.0 & 6.4 & 10.6 \\
\hline G6 & 203 & 0.1 & 0.1463 & 0.0010 & 1.4820 & 0.0149 & 0.0703 & 0.0004 & 936.0 & 12.5 & 880.4 & 16.7 & 923.0 & 16.1 & 4.6 & 5.9 \\
\hline G7 & 51 & 0.5 & 0.1318 & 0.0010 & 1.1153 & 0.0187 & 0.0662 & 0.0005 & 811.1 & 14.6 & 798.0 & 16.5 & 760.7 & 17.7 & -4.9 & 1.6 \\
\hline G8 & 60 & 0.4 & 0.1204 & 0.0009 & 1.0229 & 0.0156 & 0.0648 & 0.0005 & 766.9 & 14.0 & 732.9 & 15.6 & 715.4 & 16.8 & -2.4 & 4.4 \\
\hline G9 & 34 & 0.2 & 0.0144 & 0.0001 & 0.0979 & 0.0012 & 0.0484 & 0.0005 & 120.8 & 7.8 & 92.4 & 6.9 & 94.9 & 7.8 & 2.6 & 23.5 \\
\hline G10 & 23 & 0.8 & 0.1353 & 0.0012 & 1.2157 & 0.0357 & 0.0664 & 0.0008 & 817.4 & 19.7 & 818.2 & 18.8 & 807.8 & 22.7 & -1.3 & -0.1 \\
\hline G11 & 23 & 0.5 & 0.1377 & 0.0012 & 1.2712 & 0.0374 & 0.0673 & 0.0009 & 848.0 & 20.0 & 831.4 & 19.0 & 832.9 & 22.9 & 0.2 & 2.0 \\
\hline G12 & 110 & 1.1 & 0.1299 & 0.0010 & 1.1742 & 0.0184 & 0.0677 & 0.0005 & 859.7 & 14.4 & 787.1 & 16.3 & 788.6 & 17.3 & 0.2 & 8.4 \\
\hline G13 & 47 & 0.1 & 0.0326 & 0.0003 & 0.2506 & 0.0035 & 0.0545 & 0.0006 & 390.5 & 12.0 & 206.6 & 8.6 & 227.0 & 10.6 & 9.0 & 47.1 \\
\hline G14 & 32 & 0.7 & 0.1264 & 0.0010 & 1.1010 & 0.0228 & 0.0656 & 0.0006 & 793.0 & 16.2 & 767.5 & 16.8 & 753.9 & 19.0 & -1.8 & 3.2 \\
\hline G15 & 25 & 0.7 & 0.1731 & 0.0017 & 1.9176 & 0.0721 & 0.0838 & 0.0012 & 1286.8 & 25.7 & 1029.3 & 23.6 & 1087.2 & 28.2 & 5.3 & 20.0 \\
\hline G16 & 12 & 0.8 & 0.0161 & 0.0002 & 0.1037 & 0.0023 & 0.0482 & 0.0009 & 107.6 & 9.5 & 103.0 & 7.5 & 100.2 & 9.8 & -2.7 & 4.3 \\
\hline G17 & 48 & 0.2 & 0.0867 & 0.0007 & 0.6878 & 0.0101 & 0.0580 & 0.0005 & 529.0 & 12.5 & 536.2 & 13.3 & 531.4 & 14.9 & -0.9 & -1.4 \\
\hline G18 & 109 & 0.2 & 0.0463 & 0.0003 & 0.3653 & 0.0035 & 0.0560 & 0.0004 & 452.4 & 10.3 & 291.5 & 9.7 & 316.2 & 10.8 & 7.8 & 35.6 \\
\hline G19 & 2 & 1.0 & 0.0125 & 0.0003 & 0.0872 & 0.0055 & 0.0525 & 0.0031 & 306.8 & 36.5 & 79.8 & 8.9 & 84.9 & 16.0 & 5.9 & 74.0 \\
\hline G20 & 70 & 0.2 & 0.1150 & 0.0009 & 0.9719 & 0.0142 & 0.0630 & 0.0005 & 707.2 & 13.4 & 701.8 & 15.5 & 689.5 & 16.5 & -1.8 & 0.8 \\
\hline $\mathrm{G} 21$ & 30 & 0.4 & 0.0150 & 0.0001 & 0.1053 & 0.0016 & 0.0493 & 0.0006 & 163.5 & 9.4 & 96.2 & 7.1 & 101.7 & 8.4 & 5.4 & 41.2 \\
\hline $\mathrm{G} 22$ & 34 & 0.9 & 0.1264 & 0.0011 & 1.1170 & 0.0255 & 0.0656 & 0.0007 & 793.0 & 17.4 & 767.2 & 17.5 & 761.5 & 20.3 & -0.7 & 3.2 \\
\hline G23 & 47 & 0.5 & 0.0359 & 0.0003 & 0.2773 & 0.0052 & 0.0547 & 0.0007 & 398.3 & 14.3 & 227.2 & 9.3 & 248.5 & 12.4 & 8.6 & 43.0 \\
\hline G24 & 3 & 1.2 & 0.0136 & 0.0003 & 0.0989 & 0.0056 & 0.0539 & 0.0028 & 368.1 & 37.2 & 86.8 & 8.9 & 95.7 & 15.7 & 9.4 & 76.4 \\
\hline $\mathrm{G} 25$ & 105 & 0.1 & 0.1489 & 0.0012 & 1.3667 & 0.0231 & 0.0699 & 0.0006 & 924.5 & 15.2 & 894.6 & 18.5 & 874.8 & 18.9 & -2.3 & 3.2 \\
\hline G26 & 234 & 0.2 & 0.3258 & 0.0024 & 4.9614 & 0.0651 & 0.1120 & 0.0007 & 1832.0 & 15.3 & 1817.8 & 29.1 & 1812.8 & 21.5 & -0.3 & 0.8 \\
\hline G27 & 21 & 0.7 & 0.0164 & 0.0002 & 0.1093 & 0.0022 & 0.0471 & 0.0008 & 55.8 & 7.3 & 104.9 & 7.4 & 105.4 & 9.3 & 0.4 & -88.0 \\
\hline G28 & 1016 & 0.1 & 0.4176 & 0.0031 & 8.5687 & 0.0841 & 0.1458 & 0.0008 & 2297.5 & 15.2 & 2249.5 & 33.6 & 2293.2 & 22.1 & 1.9 & 2.1 \\
\hline G29 & 100 & 0.5 & 0.3420 & 0.0027 & 5.4574 & 0.1103 & 0.1177 & 0.0008 & 1920.8 & 17.2 & 1896.2 & 31.3 & 1893.9 & 23.4 & -0.1 & 1.3 \\
\hline G30 & 35 & 0.4 & 0.0922 & 0.0008 & 0.8125 & 0.0163 & 0.0643 & 0.0007 & 751.2 & 16.9 & 568.7 & 14.7 & 603.9 & 18.0 & 5.8 & 24.3 \\
\hline G31 & 181 & 0.0 & 0.1427 & 0.0011 & 1.3667 & 0.0175 & 0.0684 & 0.0005 & 881.9 & 13.4 & 859.8 & 17.9 & 874.8 & 17.4 & 1.7 & 2.5 \\
\hline G32 & 269 & 0.3 & 0.3211 & 0.0025 & 4.7565 & 0.0608 & 0.1102 & 0.0007 & 1803.0 & 15.4 & 1795.1 & 29.6 & 1777.3 & 21.8 & -1.0 & 0.4 \\
\hline G33 & 79 & 0.5 & 0.2472 & 0.0020 & 2.9579 & 0.0543 & 0.0911 & 0.0007 & 1448.3 & 16.8 & 1424.0 & 26.1 & 1396.9 & 22.2 & -1.9 & 1.7 \\
\hline G34 & 17 & 0.4 & 0.0108 & 0.0001 & 0.0708 & 0.0014 & 0.0474 & 0.0009 & 70.9 & 8.0 & 69.1 & 6.9 & 69.4 & 8.3 & 0.4 & 2.6 \\
\hline G35 & 3 & 0.6 & 0.0144 & 0.0002 & 0.0938 & 0.0038 & 0.0480 & 0.0018 & 100.7 & 12.5 & 91.9 & 8.2 & 91.1 & 12.4 & -0.9 & 8.8 \\
\hline G36 & 8 & 0.9 & 0.0076 & 0.0001 & 0.0527 & 0.0014 & 0.0493 & 0.0012 & 161.6 & 12.7 & 48.9 & 6.6 & 52.1 & 8.2 & 6.2 & 69.8 \\
\hline G37 & 52 & 0.2 & 0.0343 & 0.0003 & 0.2454 & 0.0032 & 0.0512 & 0.0005 & 248.0 & 9.6 & 217.5 & 9.0 & 222.8 & 10.4 & 2.4 & 12.3 \\
\hline G38 & 21 & 0.1 & 0.0305 & 0.0003 & 0.2000 & 0.0035 & 0.0500 & 0.0007 & 193.6 & 10.1 & 193.6 & 8.9 & 185.1 & 11.0 & -4.6 & 0.0 \\
\hline G39 & 131 & 0.4 & 0.1505 & 0.0012 & 1.4616 & 0.0213 & 0.0701 & 0.0005 & 931.9 & 14.5 & 903.8 & 19.2 & 914.7 & 18.7 & 1.2 & 3.0 \\
\hline G40 & 56 & 0.1 & 0.0550 & 0.0005 & 0.4413 & 0.0071 & 0.0571 & 0.0006 & 494.6 & 13.6 & 345.3 & 11.2 & 371.2 & 13.7 & 7.0 & 30.2 \\
\hline G41 & 45 & 0.2 & 0.1180 & 0.0011 & 1.0086 & 0.0233 & 0.0665 & 0.0008 & 822.2 & 18.5 & 718.9 & 17.8 & 708.2 & 21.0 & -1.5 & 12.6 \\
\hline G42 & 44 & 0.5 & 0.1646 & 0.0015 & 1.5836 & 0.0366 & 0.0748 & 0.0008 & 1061.8 & 18.9 & 982.3 & 21.8 & 963.8 & 22.8 & -1.9 & 7.5 \\
\hline G43 & 59 & 0.4 & 0.0920 & 0.0008 & 0.6954 & 0.0109 & 0.0592 & 0.0005 & 573.0 & 13.5 & 567.4 & 14.7 & 536.0 & 16.3 & -5.8 & 1.0 \\
\hline G44 & 12 & 0.4 & 0.0104 & 0.0001 & 0.0693 & 0.0014 & 0.0479 & 0.0009 & 93.8 & 8.7 & 66.8 & 6.8 & 68.0 & 8.1 & 1.9 & 28.9 \\
\hline G45 & 156 & 0.0 & 0.0488 & 0.0004 & 0.3714 & 0.0041 & 0.0542 & 0.0004 & 377.3 & 10.0 & 307.4 & 10.4 & 320.7 & 11.4 & 4.1 & 18.5 \\
\hline G46 & 31 & 0.8 & 0.1380 & 0.0013 & 1.2413 & 0.0323 & 0.0685 & 0.0008 & 883.1 & 19.8 & 833.1 & 20.3 & 819.5 & 23.2 & -1.7 & 5.7 \\
\hline & & & & & & & & & & & & & & & & \\
\hline
\end{tabular}




\begin{tabular}{|c|c|c|c|c|c|c|c|c|c|c|c|c|c|c|c|c|}
\hline G48 & 244 & 0.5 & 0.6087 & 0.0051 & 21.0959 & 0.3371 & 0.2719 & 0.0017 & 3316.7 & 17.9 & 3064.7 & 46.6 & 3143.0 & 26.0 & 2.5 & 7.6 \\
\hline G49 & 127 & 0.1 & 0.0475 & 0.0004 & 0.3652 & 0.0043 & 0.0551 & 0.0004 & 415.5 & 10.6 & 299.3 & 10.5 & 316.1 & 11.6 & 5.3 & 28.0 \\
\hline G50 & 5 & 0.1 & 0.0136 & 0.0002 & 0.0956 & 0.0030 & 0.0517 & 0.0014 & 271.3 & 18.8 & 87.1 & 7.8 & 92.7 & 11.0 & 6.1 & 67.9 \\
\hline G51 & 23 & 1.1 & 0.1289 & 0.0013 & 1.1284 & 0.0317 & 0.0672 & 0.0009 & 843.7 & 21.2 & 781.4 & 20.4 & 767.0 & 24.3 & -1.9 & 7.4 \\
\hline G52 & 120 & 0.8 & 0.4614 & 0.0042 & 9.5689 & 0.1998 & 0.1611 & 0.0012 & 2467.2 & 19.1 & 2445.7 & 42.1 & 2394.2 & 26.9 & -2.2 & 0.9 \\
\hline G53 & 67 & 0.6 & 0.1665 & 0.0015 & 1.5935 & 0.0283 & 0.0717 & 0.0006 & 978.0 & 16.3 & 992.5 & 22.3 & 967.7 & 21.4 & -2.6 & -1.5 \\
\hline G54 & 115 & 0.5 & 0.1808 & 0.0016 & 1.7726 & 0.0277 & 0.0732 & 0.0006 & 1018.7 & 15.5 & 1071.5 & 23.3 & 1035.5 & 21.1 & -3.5 & -5.2 \\
\hline G55 & 353 & 0.1 & 0.1324 & 0.0012 & 1.2237 & 0.0148 & 0.0667 & 0.0005 & 827.2 & 13.3 & 801.3 & 18.8 & 811.5 & 18.0 & 1.2 & 3.1 \\
\hline G56 & 855 & 0.3 & 0.4843 & 0.0043 & 10.6701 & 0.1377 & 0.1590 & 0.0011 & 2445.3 & 17.9 & 2545.8 & 42.8 & 2494.8 & 26.2 & -2.0 & -4.1 \\
\hline G57 & 14 & 0.6 & 0.0161 & 0.0002 & 0.1179 & 0.0024 & 0.0539 & 0.0009 & 366.5 & 15.8 & 102.7 & 7.6 & 113.2 & 9.8 & 9.2 & 72.0 \\
\hline G58 & 69 & 0.5 & 0.1066 & 0.0010 & 0.8543 & 0.0139 & 0.0608 & 0.0006 & 633.2 & 14.0 & 652.8 & 17.0 & 627.0 & 17.9 & -4.1 & -3.1 \\
\hline G59 & 55 & 0.5 & 0.1517 & 0.0014 & 1.3676 & 0.0250 & 0.0695 & 0.0006 & 914.5 & 16.4 & 910.7 & 21.5 & 875.1 & 21.2 & -4.1 & 0.4 \\
\hline G60 & 350 & 0.3 & 0.4328 & 0.0039 & 8.7192 & 0.1294 & 0.1454 & 0.0010 & 2292.4 & 18.2 & 2318.3 & 40.9 & 2309.1 & 26.4 & -0.4 & -1.1 \\
\hline G61 & 84 & 0.6 & 0.4552 & 0.0043 & 9.6289 & 0.2122 & 0.1620 & 0.0013 & 2476.9 & 19.8 & 2418.5 & 43.5 & 2399.9 & 28.0 & -0.8 & 2.4 \\
\hline G62 & 59 & 0.2 & 0.0908 & 0.0009 & 0.7463 & 0.0119 & 0.0615 & 0.0006 & 655.4 & 14.1 & 560.1 & 15.5 & 566.1 & 16.9 & 1.1 & 14.5 \\
\hline G63 & 96 & 0.3 & 0.3992 & 0.0038 & 6.9336 & 0.1414 & 0.1298 & 0.0010 & 2095.4 & 19.3 & 2165.2 & 40.2 & 2103.0 & 27.3 & -3.0 & -3.3 \\
\hline G64 & 16 & 0.5 & 0.1061 & 0.0011 & 0.8484 & 0.0228 & 0.0607 & 0.0009 & 628.3 & 18.9 & 650.1 & 18.5 & 623.7 & 22.5 & -4.2 & -3.5 \\
\hline G65 & 6 & 1.2 & 0.1124 & 0.0015 & 0.8752 & 0.0408 & 0.0630 & 0.0016 & 708.6 & 30.7 & 686.8 & 23.2 & 638.4 & 33.8 & -7.6 & 3.1 \\
\hline G66 & 395 & 0.2 & 0.1377 & 0.0013 & 1.2672 & 0.0170 & 0.0662 & 0.0005 & 811.8 & 13.8 & 831.5 & 20.0 & 831.1 & 18.9 & 0.0 & -2.4 \\
\hline G67 & 20 & 1.1 & 0.1367 & 0.0015 & 1.1526 & 0.0329 & 0.0654 & 0.0009 & 785.9 & 20.9 & 825.8 & 22.3 & 778.5 & 25.4 & -6.1 & -5.1 \\
\hline G68 & 49 & 0.3 & 0.0799 & 0.0008 & 0.6413 & 0.0110 & 0.0596 & 0.0006 & 588.7 & 14.2 & 495.6 & 14.9 & 503.1 & 16.7 & 1.5 & 15.8 \\
\hline G69 & 166 & 0.6 & 0.4425 & 0.0044 & 9.3455 & 0.2036 & 0.1644 & 0.0013 & 2501.4 & 20.6 & 2361.8 & 44.8 & 2372.5 & 29.2 & 0.5 & 5.6 \\
\hline G70 & 83 & 0.1 & 0.1525 & 0.0015 & 1.3780 & 0.0258 & 0.0690 & 0.0006 & 897.8 & 16.5 & 915.2 & 22.6 & 879.6 & 21.9 & -4.0 & -1.9 \\
\hline G71 & 49 & 0.5 & 0.1466 & 0.0015 & 1.2111 & 0.0262 & 0.0647 & 0.0007 & 764.3 & 17.1 & 881.7 & 22.6 & 805.7 & 22.7 & -9.4 & -15.4 \\
\hline G72 & 152 & 0.5 & 0.1380 & 0.0014 & 1.3429 & 0.0230 & 0.0701 & 0.0006 & 930.1 & 16.1 & 833.4 & 21.1 & 864.5 & 20.9 & 3.6 & 10.4 \\
\hline G73 & 33 & 0.4 & 0.1473 & 0.0016 & 1.2295 & 0.0300 & 0.0643 & 0.0008 & 750.9 & 18.2 & 885.6 & 23.1 & 814.1 & 23.9 & -8.8 & -18.0 \\
\hline G74 & 32 & 0.0 & 0.1563 & 0.0017 & 1.4005 & 0.0320 & 0.0693 & 0.0008 & 906.2 & 18.6 & 936.0 & 24.0 & 889.2 & 24.1 & -5.3 & -3.3 \\
\hline G75 & 12 & 0.6 & 0.0688 & 0.0008 & 0.5250 & 0.0146 & 0.0572 & 0.0010 & 499.3 & 18.9 & 428.9 & 15.3 & 428.5 & 20.2 & -0.1 & 14.1 \\
\hline G76 & 48 & 0.2 & 0.0357 & 0.0004 & 0.2463 & 0.0043 & 0.0493 & 0.0005 & 162.1 & 8.6 & 226.1 & 10.2 & 223.5 & 11.4 & -1.2 & -39.5 \\
\hline G77 & 61 & 0.5 & 0.1797 & 0.0019 & 1.7085 & 0.0383 & 0.0732 & 0.0008 & 1018.7 & 18.8 & 1065.2 & 26.6 & 1011.7 & 25.0 & -5.3 & -4.6 \\
\hline G78 & 32 & 0.1 & 0.1041 & 0.0011 & 0.7810 & 0.0173 & 0.0577 & 0.0007 & 518.0 & 15.2 & 638.2 & 18.8 & 586.0 & 20.4 & -8.9 & -23.2 \\
\hline G79 & 2 & 0.8 & 0.0155 & 0.0003 & 0.1056 & 0.0053 & 0.0554 & 0.0025 & 428.0 & 37.3 & 98.8 & 9.3 & 102.0 & 16.1 & 3.1 & 76.9 \\
\hline G80 & 117 & 0.2 & 0.1552 & 0.0017 & 1.7539 & 0.0392 & 0.0830 & 0.0009 & 1269.9 & 20.5 & 929.9 & 24.5 & 1028.6 & 25.1 & 9.6 & 26.8 \\
\hline G81 & 86 & 0.2 & 0.0370 & 0.0003 & 0.2533 & 0.0053 & 0.0498 & 0.0025 & 185.2 & 22.6 & 233.9 & 9.2 & 229.2 & 26.6 & -2.0 & -26.3 \\
\hline G82 & 438 & 0.2 & 0.1594 & 0.0017 & 1.5177 & 0.0392 & 0.0692 & 0.0009 & 903.2 & 20.4 & 953.2 & 24.4 & 937.5 & 25.6 & -1.7 & -5.5 \\
\hline G83 & 11 & 0.6 & 0.0096 & 0.0004 & 0.0658 & 0.0047 & 0.0494 & 0.0006 & 165.4 & 8.9 & 61.8 & 10.6 & 64.7 & 10.9 & 4.6 & 62.7 \\
\hline G84 & 3 & 0.9 & 0.0129 & 0.0001 & 0.0819 & 0.0020 & 0.0495 & 0.0013 & 170.2 & 13.5 & 82.3 & 7.1 & 80.0 & 10.0 & -2.9 & 51.6 \\
\hline G85 & 20 & 0.6 & 0.0280 & 0.0020 & 0.1910 & 0.0364 & 0.0507 & 0.0007 & 226.8 & 11.4 & 178.0 & 30.4 & 177.5 & 29.6 & -0.3 & 21.5 \\
\hline G86 & 60 & 0.5 & 0.1526 & 0.0002 & 1.2767 & 0.0035 & 0.0651 & 0.0019 & 778.2 & 37.7 & 915.3 & 7.9 & 835.4 & 40.3 & -9.6 & -17.6 \\
\hline
\end{tabular}

SAMPLE: MAK 2-4

\begin{tabular}{|c|c|c|c|c|c|c|c|c|c|c|c|c|c|c|c|c|}
\hline \multirow[b]{2}{*}{ Grain } & \multirow[b]{2}{*}{$\begin{array}{c}\mathbf{U} \\
(\mathbf{p p m})\end{array}$} & \multirow[b]{2}{*}{$\begin{array}{c}\text { Atomic } \\
\text { Th/ U }\end{array}$} & \multirow[b]{2}{*}{$\frac{206 \mathrm{~Pb}}{238 \mathrm{U}}$} & \multirow[b]{2}{*}{$\begin{array}{c} \pm 1 \\
\text { sigma }\end{array}$} & \multirow[b]{2}{*}{$\frac{207 P b}{235 U}$} & \multirow[b]{2}{*}{$\begin{array}{c} \pm 1 \\
\text { sigma }\end{array}$} & \multirow[b]{2}{*}{$\frac{207 \mathrm{~Pb}}{206 \mathrm{~Pb}}$} & \multirow[b]{2}{*}{$\begin{array}{c} \pm 1 \\
\text { sigma }\end{array}$} & \multicolumn{6}{|c|}{ Apparent Ages (Ma) } & \multirow{2}{*}{$\begin{array}{c}\begin{array}{c}\text { Corr.\% } \\
\text { discordant }\end{array} \\
\frac{206 / 238}{207 / 235}\end{array}$} & \multirow{2}{*}{$\begin{array}{c}\begin{array}{c}\text { Corr.\% } \\
\text { discordant }\end{array} \\
\frac{207 / 206}{206 / 238} \\
\end{array}$} \\
\hline & & & & & & & & & $\frac{207 \mathrm{~Pb}}{206 \mathrm{~Pb}}$ & $\begin{array}{c} \pm 2 \\
\text { sigma }\end{array}$ & $\frac{206 \mathrm{~Pb}}{238 \mathrm{U}}$ & $\begin{array}{c} \pm 2 \\
\text { sigma }\end{array}$ & $\frac{207 P b}{235 U}$ & $\begin{array}{c} \pm 2 \\
\text { sigma }\end{array}$ & & \\
\hline$\overline{\mathrm{G} 1}$ & 4419 & 0.2 & 0.3757 & 0.0032 & $\bar{~} 5.57204$ & (0.0753 & 0.11409 & 0.0013 & $=1865.6$ & 24.3 & 2056.2 & 34.1 & "1911.8 & 29.4 & -7.6 & $\begin{array}{l}-7.0 \\
\end{array}$ \\
\hline G2 & 657 & 1.2 & 0.0896 & 0.0008 & 0.71589 & 0.0119 & 0.0561 & 0.0008 & 456.3 & 15.4 & 552.9 & 13.7 & 548.2 & 18.8 & -0.9 & -97.5 \\
\hline G3 & 133 & 0.6 & 0.1132 & 0.001 & 0.90329 & 0.0204 & 0.06074 & 0.0011 & 630.0 & 21.6 & 691.1 & 16.5 & 653.5 & 24.6 & -5.8 & -33.4 \\
\hline G4 & 149 & 0.3 & 0.4561 & 0.0039 & 9.02856 & 0.1434 & 0.15375 & 0.0018 & 2388.1 & 26.2 & 2422.3 & 39.1 & 2340.9 & 31.3 & -3.5 & -2.2 \\
\hline G5 & 452 & 0.4 & 0.091 & 0.0008 & 0.70264 & 0.0107 & 0.05761 & 0.0008 & 515.0 & 15.9 & 561.6 & 13.9 & 540.4 & 18.6 & -3.9 & -29.8 \\
\hline
\end{tabular}




\begin{tabular}{|c|c|c|c|c|c|c|c|c|c|c|c|c|c|c|c|c|}
\hline G6 & 337 & 0.8 & 0.0904 & 0.0008 & 0.69156 & 0.011 & 0.05788 & 0.0008 & 525.2 & 16.5 & 558.1 & 13.9 & 533.7 & 19.0 & -4.6 & -42.5 \\
\hline G7 & 181 & 1.0 & 0.3407 & 0.003 & 4.94478 & 0.0862 & 0.11389 & 0.0014 & 1862.4 & 26.0 & 1890.0 & 33.4 & 1809.9 & 30.8 & -4.4 & -13.9 \\
\hline G8 & 125 & 0.6 & 0.0166 & 0.0002 & 0.12264 & 0.0058 & 0.05423 & 0.0025 & 380.6 & 33.6 & 105.9 & 7.2 & 117.5 & 15.2 & 9.9 & -75.6 \\
\hline G9 & 1145 & 0.6 & 0.0065 & 7E-05 & 0.04239 & 0.0012 & 0.04612 & 0.0013 & 3.9 & 4.8 & 42.0 & 5.5 & 42.2 & 7.0 & 0.5 & -139.9 \\
\hline G10 & 69 & 0.9 & 0.0115 & 0.0002 & 0.07179 & 0.0065 & 0.04808 & 0.0043 & 103.2 & 22.2 & 73.4 & 7.0 & 70.4 & 17.7 & -4.3 & -23.0 \\
\hline G11 & 363 & 0.4 & 0.0187 & 0.0002 & 0.12658 & 0.0043 & 0.05364 & 0.0018 & 356.0 & 24.4 & 119.1 & 7.2 & 121.0 & 13.1 & 1.6 & -13.0 \\
\hline G12 & 677 & 0.4 & 0.0138 & 0.0001 & 0.09429 & 0.0021 & 0.04843 & 0.0011 & 120.3 & 9.7 & 88.2 & 6.2 & 91.5 & 8.7 & 3.6 & -44.8 \\
\hline G13 & 328 & 0.4 & 0.0075 & 0.0001 & 0.05303 & 0.0025 & 0.05238 & 0.0024 & 302.0 & 28.6 & 48.3 & 5.8 & 52.5 & 9.6 & 7.9 & -25.2 \\
\hline G14 & 622 & 0.6 & 0.0139 & 0.0001 & 0.09189 & 0.0027 & 0.04734 & 0.0013 & 66.4 & 8.2 & 89.1 & 6.3 & 89.3 & 9.6 & 0.2 & -76.4 \\
\hline G15 & 382 & 0.5 & 0.0181 & 0.0002 & 0.11859 & 0.0032 & 0.05029 & 0.0013 & 208.4 & 14.4 & 115.4 & 6.8 & 113.8 & 10.9 & -1.4 & -34.2 \\
\hline G16 & 407 & 0.2 & 0.1847 & 0.0017 & 1.80741 & 0.0296 & 0.07573 & 0.001 & 1087.9 & 22.9 & 1092.7 & 23.2 & 1048.1 & 26.7 & -4.3 & 1.6 \\
\hline G17 & 277 & 1.3 & 0.1074 & 0.001 & 0.83051 & 0.0153 & 0.05946 & 0.0009 & 584.0 & 18.6 & 657.7 & 16.3 & 613.9 & 22.0 & -7.1 & -99.4 \\
\hline G18 & 193 & 0.7 & 0.0115 & 0.0002 & 0.08366 & 0.0039 & 0.0553 & 0.0025 & 424.4 & 35.9 & 73.6 & 6.5 & 81.6 & 12.2 & 9.8 & -58.9 \\
\hline G19 & 203 & 0.4 & 0.5321 & 0.005 & 12.39775 & 0.2119 & 0.17969 & 0.0024 & 2650.0 & 29.2 & 2750.3 & 46.3 & 2635.0 & 34.9 & -4.4 & -4.1 \\
\hline G20 & 1056 & 0.5 & 0.0325 & 0.0003 & 0.23471 & 0.0039 & 0.05179 & 0.0008 & 276.1 & 12.2 & 205.9 & 8.4 & 214.1 & 11.6 & 3.8 & -42.3 \\
\hline G21 & 169 & 0.8 & 0.0179 & 0.0002 & 0.1208 & 0.0052 & 0.05118 & 0.0021 & 248.9 & 23.0 & 114.1 & 7.3 & 115.8 & 14.5 & 1.5 & -43.4 \\
\hline G22 & 635 & 0.6 & 0.0117 & 0.0001 & 0.08119 & 0.0022 & 0.05016 & 0.0013 & 202.4 & 14.0 & 75.1 & 6.1 & 79.3 & 8.8 & 5.2 & -62.0 \\
\hline G23 & 847 & 0.4 & 0.0167 & 0.0002 & 0.11556 & 0.0024 & 0.04952 & 0.001 & 172.5 & 10.9 & 106.4 & 6.7 & 111.0 & 9.2 & 4.1 & -31.1 \\
\hline G24 & 1046 & 0.5 & 0.0169 & 0.0002 & 0.11521 & 0.0024 & 0.04825 & 0.001 & 111.5 & 8.8 & 108.0 & 6.7 & 110.7 & 9.1 & 2.4 & -56.7 \\
\hline G25 & 588 & 0.2 & 0.3377 & 0.0032 & 5.2327 & 0.0835 & 0.11159 & 0.0015 & 1825.5 & 27.8 & 1875.7 & 35.5 & 1858.0 & 33.0 & -1.0 & -3.6 \\
\hline G26 & 459 & 1.5 & 0.0948 & 0.0009 & 0.81132 & 0.0145 & 0.06215 & 0.001 & 679.3 & 20.0 & 583.9 & 15.4 & 603.2 & 21.3 & 3.2 & -272.9 \\
\hline G27 & 250 & 0.6 & 0.0182 & 0.0002 & 0.12449 & 0.0039 & 0.05236 & 0.0016 & 301.2 & 20.2 & 116.2 & 7.1 & 119.1 & 12.1 & 2.5 & -14.7 \\
\hline G28 & 115 & 0.7 & 0.0175 & 0.0003 & 0.12165 & 0.0103 & 0.05406 & 0.0044 & 373.5 & 55.4 & 111.8 & 8.9 & 116.6 & 24.2 & 4.1 & -56.1 \\
\hline G29 & 9 & 0.6 & 0.4373 & 0.0086 & 7.14769 & 0.7545 & 0.17314 & 0.0069 & 2588.2 & 78.6 & 2338.7 & 81.8 & 2130.0 & 86.5 & -9.8 & 0.2 \\
\hline G30 & 269 & 0.3 & 0.1821 & 0.0018 & 1.75492 & 0.0341 & 0.07406 & 0.0012 & 1043.1 & 25.2 & 1078.7 & 24.1 & 1029.0 & 29.1 & -4.8 & -8.8 \\
\hline G31 & 99 & 0.4 & 0.1416 & 0.0016 & 1.30613 & 0.0446 & 0.07149 & 0.0017 & 971.5 & 35.0 & 853.5 & 22.4 & 848.4 & 36.2 & -0.6 & -11.2 \\
\hline G32 & 631 & 0.1 & 0.1821 & 0.0018 & 2.06782 & 0.0396 & 0.08012 & 0.0013 & 1199.9 & 26.5 & 1078.3 & 24.2 & 1138.2 & 29.6 & 5.3 & 4.6 \\
\hline G33 & 638 & 0.4 & 0.0204 & 0.0002 & 0.14206 & 0.0045 & 0.05013 & 0.0015 & 201.0 & 15.4 & 129.9 & 7.5 & 134.9 & 12.5 & 3.7 & -15.0 \\
\hline G34 & 771 & 0.3 & 0.0173 & 0.0002 & 0.11967 & 0.0029 & 0.04947 & 0.0011 & 170.2 & 11.5 & 110.8 & 6.8 & 114.8 & 9.8 & 3.5 & -13.1 \\
\hline G35 & 119 & 0.9 & 0.1394 & 0.0015 & 1.16641 & 0.0303 & 0.06469 & 0.0013 & 764.3 & 26.1 & 841.1 & 21.2 & 785.0 & 30.1 & -7.2 & -33.5 \\
\hline G36 & 343 & 0.4 & 0.0138 & 0.0002 & 0.09884 & 0.0035 & 0.05533 & 0.0019 & 425.6 & 27.8 & 88.3 & 6.7 & 95.7 & 11.4 & 7.7 & -6.4 \\
\hline G37 & 203 & 0.3 & 0.585 & 0.006 & 14.90634 & 0.3437 & 0.19452 & 0.0032 & 2780.8 & 35.6 & 2969.2 & 53.3 & 2809.3 & 41.4 & -5.7 & -5.3 \\
\hline G38 & 365 & 0.7 & 0.0169 & 0.0002 & 0.11846 & 0.0042 & 0.05187 & 0.0017 & 279.7 & 20.9 & 108.2 & 7.1 & 113.7 & 12.3 & 4.8 & -36.8 \\
\hline G39 & 2193 & 0.5 & 0.0071 & $8 \mathrm{E}-05$ & 0.05029 & 0.0012 & 0.05177 & 0.0011 & 275.3 & 15.2 & 45.4 & 5.6 & 49.8 & 7.0 & 9.0 & -22.8 \\
\hline G40 & 235 & 0.4 & 0.0951 & 0.001 & 0.78975 & 0.0205 & 0.0639 & 0.0013 & 738.3 & 26.6 & 585.7 & 16.7 & 591.0 & 26.4 & 0.9 & -7.8 \\
\hline G41 & 469 & 0.1 & 0.1031 & 0.0011 & 0.80367 & 0.0191 & 0.0584 & 0.0011 & 544.8 & 20.9 & 632.6 & 17.3 & 598.9 & 24.9 & -5.6 & -7.6 \\
\hline G42 & 248 & 0.4 & 0.018 & 0.0002 & 0.11677 & 0.0047 & 0.04969 & 0.0019 & 180.5 & 16.9 & 115.0 & 7.5 & 112.1 & 13.4 & -2.6 & -16.0 \\
\hline G43 & 187 & 0.1 & 0.3593 & 0.0039 & 5.11993 & 0.1327 & 0.11 & 0.0021 & 1799.4 & 36.6 & 1978.9 & 41.1 & 1839.4 & 42.0 & -7.6 & -5.3 \\
\hline G44 & 71 & 0.6 & 0.143 & 0.0018 & 1.26515 & 0.0578 & 0.06546 & 0.0021 & 789.2 & 39.9 & 861.3 & 25.0 & 830.2 & 44.2 & -3.7 & -26.4 \\
\hline G45 & 316 & 0.7 & 0.0152 & 0.0002 & 0.09398 & 0.0038 & 0.0479 & 0.0018 & 94.3 & 11.4 & 97.2 & 7.0 & 91.2 & 11.9 & -6.6 & -44.1 \\
\hline G46 & 512 & 0.4 & 0.1914 & 0.0021 & 1.99474 & 0.0496 & 0.07581 & 0.0015 & 1090.1 & 30.8 & 1129.0 & 26.8 & 1113.7 & 34.9 & -1.4 & -12.7 \\
\hline G47 & 403 & 0.4 & 0.4389 & 0.0047 & 10.0566 & 0.2488 & 0.17319 & 0.0033 & 2588.7 & 40.7 & 2345.9 & 46.8 & 2440.0 & 45.5 & 3.9 & 1.4 \\
\hline G48 & 478 & 0.7 & 0.0987 & 0.0011 & 0.79598 & 0.0205 & 0.05938 & 0.0012 & 581.1 & 22.9 & 606.9 & 17.2 & 594.6 & 25.8 & -2.1 & -35.1 \\
\hline G49 & 192 & 0.3 & 0.0977 & 0.0012 & 0.76095 & 0.0302 & 0.05801 & 0.0018 & 530.1 & 30.0 & 600.6 & 18.7 & 574.6 & 34.1 & -4.5 & -9.0 \\
\hline G50 & 110 & 0.6 & 0.1548 & 0.0018 & 1.47053 & 0.0458 & 0.07286 & 0.0017 & 1010.1 & 34.6 & 927.8 & 24.2 & 918.3 & 36.8 & -1.0 & -14.5 \\
\hline G51 & 54 & 2.0 & 0.1014 & 0.0012 & 0.77928 & 0.0304 & 0.05772 & 0.0018 & 519.1 & 29.3 & 622.8 & 19.1 & 585.1 & 34.4 & -6.4 & -155.9 \\
\hline G52 & 393 & 0.3 & 0.0077 & 0.0001 & 0.05321 & 0.0025 & 0.05227 & 0.0023 & 297.2 & 27.2 & 49.4 & 6.0 & 52.6 & 9.5 & 6.1 & -9.0 \\
\hline G53 & 172 & 0.4 & 0.0134 & 0.0002 & 0.08676 & 0.0044 & 0.0497 & 0.0023 & 181.0 & 20.1 & 86.0 & 7.0 & 84.5 & 12.9 & -1.8 & -20.3 \\
\hline G54 & 777 & 0.1 & 0.3386 & 0.0038 & 5.33023 & 0.1534 & 0.11175 & 0.0025 & 1828.1 & 42.8 & 1879.9 & 41.3 & 1873.7 & 47.5 & -0.3 & -1.9 \\
\hline G55 & 167 & 0.3 & 0.1188 & 0.0014 & 0.98002 & 0.0325 & 0.06436 & 0.0017 & 753.5 & 32.0 & 723.5 & 20.6 & 693.6 & 34.1 & -4.3 & -11.0 \\
\hline G56 & 738 & 0.7 & 0.0076 & 0.0001 & 0.05057 & 0.0026 & 0.04759 & 0.0023 & 78.9 & 11.8 & 48.6 & 6.0 & 50.1 & 9.3 & 3.1 & -66.3 \\
\hline G57 & 189 & 0.5 & 0.0985 & 0.0012 & 0.75751 & 0.0253 & 0.05996 & 0.0016 & 602.1 & 28.3 & 605.8 & 18.2 & 572.6 & 30.7 & -5.8 & -14.1 \\
\hline G58 & 99 & 0.4 & 0.0187 & 0.0003 & 0.12444 & 0.0082 & 0.05222 & 0.0032 & 295.1 & 35.9 & 119.1 & 8.5 & 119.1 & 19.8 & 0.0 & -19.9 \\
\hline G59 & 468 & 0.1 & 0.1141 & 0.0013 & 0.98479 & 0.0314 & 0.06412 & 0.0016 & 745.6 & 30.9 & 696.7 & 19.9 & 696.1 & 32.6 & -0.1 & -10.1 \\
\hline G60 & 561 & 0.2 & 0.3675 & 0.0043 & 5.99072 & 0.1923 & 0.11779 & 0.0029 & 1923.0 & 47.6 & 2017.6 & 45.0 & 1974.5 & 52.5 & -2.2 & -4.5 \\
\hline G61 & 877 & 0.1 & 0.1484 & 0.0017 & 1.4739 & 0.0479 & 0.07222 & 0.0018 & 992.1 & 36.8 & 892.0 & 24.1 & 919.7 & 38.5 & 3.0 & 5.4 \\
\hline
\end{tabular}


$\begin{array}{lcccccccc}\text { G62 } & 637 & 0.4 & 0.0182 & 0.0002 & 0.11547 & 0.0045 & 0.04586 & 0.0015 \\ \text { G63 } & 89 & 0.2 & 0.1681 & 0.0021 & 1.50182 & 0.0631 & 0.06813 & 0.0021\end{array}$ $\begin{array}{llllll}0.1681 & 0.0021 & 1.50182 & 0.0631 & 0.06813 & 0.0021 \\ 0.0374 & 0.0005 & 0.25848 & 0.0103 & 0.05213 & 0.0017 \\ 0.0201 & 0.0003 & 0.14244 & 0.006 & 0.05405 & 0.002\end{array}$

$\begin{array}{rrr}-9.7 & 3.9 & 116.5 \\ 872.5 & 40.5 & 1001.6\end{array}$

$\begin{array}{rrrrr}7.5 & 111.0 & 12.1 & -5.0 & -59.8 \\ 28.0 & 931.1 & 45.8 & -7.6 & -13.0\end{array}$ $\begin{array}{llllll}0.0201 & 0.0003 & 0.14244 & 0.006 & 0.05405 & 0.002\end{array}$ $\begin{array}{lll}291.1 & 21.2 & 236.9\end{array}$ $\begin{array}{lllllr}0.1759 & 0.0021 & 1.74208 & 0.0631 & 0.07215 & 0.002 \\ 0.0202 & 0.0003 & 0.13297 & 0.0093 & 0.05236 & 0.0034\end{array}$ $465.0 \quad 25.6 \quad 415.0$ $990.2 \quad 40.1 \quad 1044.6$ $\begin{array}{lllllll}0.0202 & 0.0003 & 0.13297 & 0.0093 & 0.05236 & 0.0034\end{array}$ $\begin{array}{lllllll}0.0158 & 0.0003 & 0.10163 & 0.0078 & 0.04954 & 0.0036\end{array}$ $\begin{array}{lllllll}0.0185 & 0.0003 & 0.11749 & 0.0058 & 0.04956 & 0.0021\end{array}$ $\begin{array}{lllllll}0.5413 & 0.0067 & 11.59052 & 0.4535 & 0.16663 & 0.0049\end{array}$ $\begin{array}{lllllll}0.1272 & 0.0016 & 1.16967 & 0.0466 & 0.06491 & 0.002\end{array}$ $\begin{array}{lllllll}0.2026 & 0.0026 & 1.98167 & 0.0852 & 0.07454 & 0.0024\end{array}$ $\begin{array}{lllllll}0.0791 & 0.0011 & 0.6911 & 0.0313 & 0.06824 & 0.0024\end{array}$ $\begin{array}{llllllll}0.0169 & 0.0002 & 0.11479 & 0.0057 & 0.04945 & 0.0021\end{array}$ $\begin{array}{llllllll}0.0197 & 0.0003 & 0.12442 & 0.0067 & 0.04908 & 0.0023\end{array}$ $\begin{array}{lllllll}0.0183 & 0.0003 & 0.11929 & 0.006 & 0.05032 & 0.0021 \\ 0.0458 & 0.0006 & 0.31705 & 0.0146 & 0.05127 & 0.0019\end{array}$ $\begin{array}{lllllll}0.0458 & 0.0006 & 0.31705 & 0.0146 & 0.05127 & 0.0019 \\ 0.0148 & 0.0002 & 0.08972 & 0.0052 & 0.04713 & 0.0024\end{array}$ $\begin{array}{lllllll}0.0148 & 0.0002 & 0.08972 & 0.0052 & 0.04713 & 0.0024\end{array}$ $\begin{array}{lllllll}0.1252 & 0.0017 & 1.04815 & 0.0488 & 0.06476 & 0.0024 \\ 0.0185 & 0.0003 & 0.11703 & 0.0063 & 0.04901 & 0.0022\end{array}$ $\begin{array}{lllllll}0.0185 & 0.0003 & 0.11703 & 0.0063 & 0.04901 & 0.0022 \\ 0.0184 & 0.0003 & 0.12526 & 0.007 & 0.05268 & 0.0025\end{array}$ $\begin{array}{lllllll}0.1261 & 0.0018 & 1.05264 & 0.0545 & 0.06351 & 0.0026\end{array}$ $\begin{array}{lll}301.2 & 38.2 & 129.0\end{array}$ $\begin{array}{lll}173.5 & 27.5 & 101.1\end{array}$ $\begin{array}{llr}174.4 & 18.4 & 118.2\end{array}$

\section{NOTES}

1 Uncertainties on individual analyses are reported at 1 sigma level and include errors on standards. Ages reported at 2 sigma

$2 \mathrm{U}$ concentration and U/Th calibrated relative to NIST SRM 612

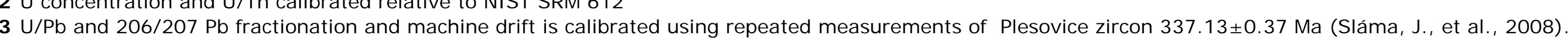

4 Best age is 206/238 for grain ages that are $\leq 10 \%$ discordant using < 1.0Ga and 206/207 for grain ages $>1.0$ Ga 
